Supporting Information

\title{
Highly Penetrable and On-Demand Oxygen Release with Tumor Activity Composite Nanosystem for Photothermal/Photodynamic Synergetic Therapy
}

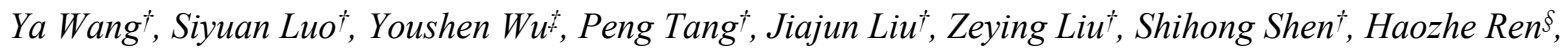

\section{Daocheng $W u^{* \dagger}$}

$\dagger$ Key Laboratory of Biomedical Information Engineering of the Ministry of Education, School of Life Science and Technology, Xi'an Jiaotong University, Xi'an 710049, P.R. China.

$\ddagger$ Department of Chemistry, School of Science, Xi’an Jiaotong University, Xi’an, 710049, P.R. China. $\S$ Health Science Center, Xi'an Jiaotong University, Xi'an, 710061 Shaanxi, P. R. China.

*Email: wudaocheng@mail.xjtu.edu.cn 


\section{A. Supplementary Methods}

Materials: Dopamine hydrochloride (99\%), hemoglobin (Hb, from bovine), Chlorin e6 (Ce6), hyaluronic acid (HA), PEI (M.W. = 10KDa), PEG (M.W. = 2KDa), 2',7'-dichlorofluorescin diacetate (DCFH-DA), 1-(3-Dimethylaminopropyl)-3-ethylcarbodiimide hydro (EDC), 4dimethylaminopyridine (DMAP), tris(hydroxymethyl)aminomethane (TRIS, 99.9\%), propidium iodide (PI), fluorescein diacetate (FDA) and 3-(4, 5-dimethyl-2-thiazolyl)-2, 5-diphenyl-2-Htetrazolium bromide (MTT) were purchased from Aladdin Co., Ltd. N,N-dimethyl-4-nitrosoaniline (RNO), 4',6-diamidino-2-phenylindole (DAPI), magnesium sulfate anhydrous, sodium chloride ( $\mathrm{NaCl})$ and 4-Formylbenzoic acid were obtained from Macklin Co., Ltd. Dulbecco's modified Eagle's medium (DMEM), trypsin, and fetal bovine serum (FBS) were obtained from Thermo Fisher Scientifc Co., Ltd. The dimethyl sulfoxide (DMSO), dichloromethane (DCM) and ethanol were obtained from Tianjin Fuyu Fine Chemical Co., Ltd.

Synthesis of Aldehyde Group Modified PEG (OHC-PEG-CHO) and Characterizations. The aldehyde group modified PEG was harvested on the basis of the previously reported method. ${ }^{23}$ In brief, $2.25 \mathrm{~g}$ of 4-carboxybenzaldehyde was added into $150 \mathrm{~mL}$ of DCM. $9.585 \mathrm{~g}$ of EDC and $0.244 \mathrm{~g}$ of DMAP were dissolved in the above solution and then stirred at $25^{\circ} \mathrm{C}$ for $6 \mathrm{~h}$. Subsequently, the solution was added by $10 \mathrm{~g}$ of PEG and stirred for $48 \mathrm{~h}$. After reaction, the crude product was concentrated, and then washed by saturated $\mathrm{NaCl}$ solution and ethyl alcohol for three times, respectively. The organic layer was collected after standing, followed by the addition of $50 \mathrm{~g}$ of anhydrous magnesium sulfate, a dehydrating agent, into the solution. After twice precipitation process with excessive ether, the filtrate was collected and dried under vacuum all night. The product was characterized by ${ }^{1} \mathrm{H}$ NMR spectra via a Bruker AV-300 NMR spectrometer (Bruker, Switzerland) in $\mathrm{CDCl}_{3}$.

Characterization of [PHC]PP@HA NPs. Hydrodynamic diameters and zeta potentials of the [PHC]PP@HA NPs at different pH (7.4 and 6.8) were measured by a Malvern instrument (ZETASIZER, NanoZS90, Malvern, Ltd., UK). High-resolution TEM ( JEM-F200, JEOL Ltd., Japan) was employed to observe the NPs morphology. In addition, the particle sizes of 200 PHC NPs, 91 [PHC]PP NPs and 76 [PHC]PP@HA NPs in the TEM images were statistically analyzed by using particle size analysis software (Nano measure 1.2). The chemical structure of the [PHC]PP@HA NPs was detected by UV-vis absorption spectroscopy (PG Instruments Ltd., China), FT-IR spectroscopy (Ettlingen, 
Germany), ${ }^{1} \mathrm{H}-\mathrm{NMR}$ spectrum (Bruker, Germany), and X-ray diffraction (XRD, Panalytical, Holland). The fluorescence spectrum was obtained by using a FluoroMax-4 Spectrofluorometer (HORIBA Jobin Yvon, USA). In vitro stabilities of [PHC]PP@HA NPs in ultrapure water, PBS (pH 7.4), glucose solution and DMEM high-glucose media containing 10\% FBS were measured. The variations of hydrodynamic diameters of [PHC]PP@HA NPs with different physiological media and times were also assessed. In addition, the stability of PHC NPs and [PHC]PP@HA NPs in serum were evaluated by measuring the UV-Vis absorption spectra within $24 \mathrm{~h}$. The thermal stability of the PHC NPs and [PHC]PP@HA NPs were also achieved by incubating samples in a $55^{\circ} \mathrm{C}$ thermostatic water bath for 4 h, and the sizes of PHC NPs and [PHC]PP@HA NPs were determined by Malvern instrument every $0.5 \mathrm{~h}$.

\section{In vitro synergistic PTT/PDT}

To investigate the synergistic effect of PTT and PDT, the viability of PC-3 cells treated by [PHC]PP@HA NPs at 670 nm or 808 nm irradiation were also measured. Briefly, the cells in 96-well plates were incubated at $37{ }^{\circ} \mathrm{C}$ under normoxia (21\% oxygen) for $24 \mathrm{~h}$. The oxygen-saturated samples were added into each well of 96-well plates and further cultured under normoxia or hypoxia (1\% oxygen) for $8 \mathrm{~h}$. Then the cells were exposed to irradiation by an $808 \mathrm{~nm}$ laser $\left(1 \mathrm{~W} \mathrm{~cm}^{-2}\right)$ for $10 \mathrm{~min}$, a $670 \mathrm{~nm}$ laser $\left(0.1 \mathrm{~W} \mathrm{~cm}^{-2}\right)$ for $15 \mathrm{~min}$, or the combination of $808 \mathrm{~nm}$ and $670 \mathrm{~nm}$ laser for $15 \mathrm{~min}$. After another $24 \mathrm{~h}$ incubation, the medium was replaced by fresh culture medium containing $0.05 \%$ MTT (200 $\mu \mathrm{L}$ per well) and kept for $4 \mathrm{~h}$. Then the medium was replaced by $200 \mu \mathrm{L}$ DMSO per well, and measured the absorbance at $490 \mathrm{~nm}$. The same method was used to evaluate the phototoxicity of [PHC]PP@HA NPs under different laser irradiation to other three cell lines, including HeLa (human cervical carcinoma cell line), A549 (human lung adenocarcinoma cell line) and PANC-1(human pancreatic carcinoma cell line).

The CI combination index (CI) value can be calculated by the following formula.

$$
\mathrm{CI}=\frac{D_{1}}{D_{\mathrm{m} 1}}+\frac{D_{1}}{D_{\mathrm{m} 2}}
$$

where D1 is the concentration when PTT and PDT produced a specified effect together, $D_{m 1}$ and $D_{m 2}$ are the doses with the same effect for a single treatment.

The LIVE/DEAD staining technique was conducted to assess the cell apoptosis induced by [PHC]PP@HA NPs. After seeding PC-3 cells into a 24-well plate at a density of $1 \times 10^{6}$ cells per well 
for $24 \mathrm{~h}$, the oxygen-saturated samples were added into the 24 -well plate and incubated with cells under hypoxia ( $1 \%$ oxygen) for another $8 \mathrm{~h}$. For laser irradiation groups, the cells exposed to $808 \mathrm{~nm}$ laser $\left(1 \mathrm{~W} \mathrm{~cm}^{-2}\right)$ for $10 \mathrm{~min}$, or $670 \mathrm{~nm}$ laser $\left(0.1 \mathrm{~W} \mathrm{~cm}^{-2}\right)$ for $15 \mathrm{~min}$, or the combination of $808 \mathrm{~nm}$ and $670 \mathrm{~nm}$ laser for $15 \mathrm{~min}$. Subsequently, the cells were stained with FDA (for live cell staining, green fluorescence) and PI (for dead cells staining, red fluorescence) in PBS for 5 min and then washed with PBS thrice before fluorescence microscope observation.

Hemolysis assay. The red blood cells (RBCs) were drawn from BALB/c mice and incubated with different amounts of [PHC]PP@HA NPs in PBS at $37{ }^{\circ} \mathrm{C}$ for $1 \mathrm{~h}$. RBCs incubated with PBS and $\mathrm{ddH}_{2} \mathrm{O}$ were employed as negative and positive controls, respectively. After incubation, the samples were centrifuged and $100 \mu \mathrm{L}$ supernatant was transferred to $96-$ well plate to examine the hemolysis. The absorbance value of supernatant was measured at $570 \mathrm{~nm}$. The calculation formula of hemolysis rate was as follows:

Hemolysis $(\%)=\frac{\text { Sample absorbance }- \text { Negative control absorbance }}{\text { Positive control absorbance }- \text { Negative control absorbance }} \times 100$ 


\section{B. Supplementary Figures}

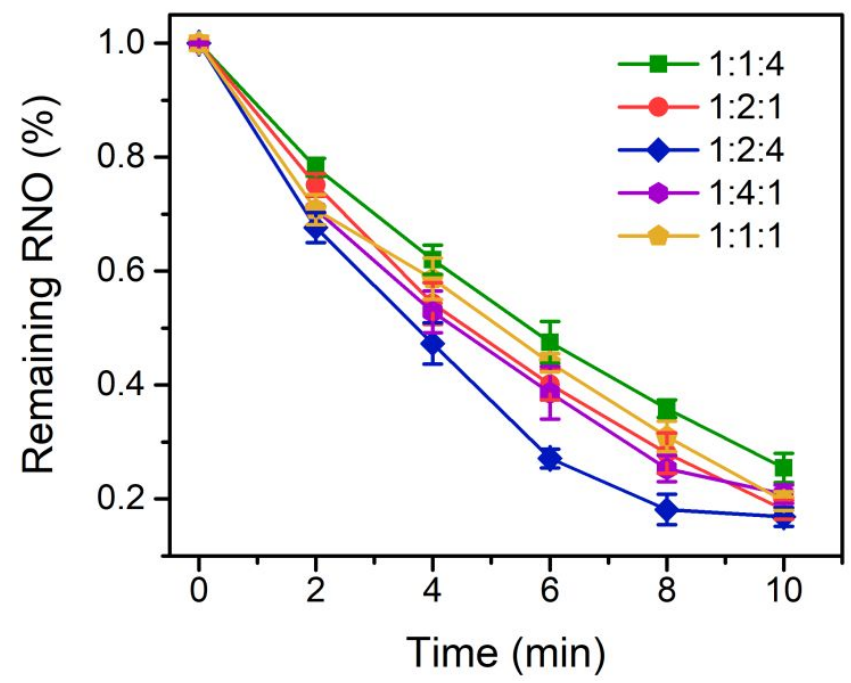

Figure S1. The ROS production efficiency of PHC NPs prepared by different PDA, $\mathrm{Hb}$ and Ce6 ratios.

The optimal ratio of PDA, $\mathrm{Hb}$ and $\mathrm{Ce} 6$ was determined by measuring the in vitro ROS production efficiency of PHC NPs with different proportions of $\mathrm{Hb}$ and Ce6. Assuming that the amount of dopamine was constant, the ROS productions of PHC NPs was compared when the ratios of PDA, Ce6 and $\mathrm{Hb}$ were 1:1:4, 1:2:4, 1:2:1, 1:4:1 and 1:1:1, respectively. As shown in Figure S1, when PDA: $\mathrm{Ce} 6: \mathrm{Hb}=1: 2: 4$, the absorbance of RNO (N,N-dimethyl-4-nitrosoaniline, a singlet oxygen indicator) decreased fastest, indicating the highest ROS generation efficiency. The reason can be that ROS production is less when the Ce6 content is low (PDA: Ce6: Hb = 1:1:4). However, the excess Ce6 cannot be completely loaded in PHC NPs under condition of high content of Ce6 (PDA: Ce6: Hb = 1:4:1), which led to less ROS production. Considering the suitable particle size of PHC NPs, the optimal ratio PDA: $\mathrm{Ce} 6: \mathrm{Hb}=1: 2: 4$ was chosen to prepare PHC NPs. 
A

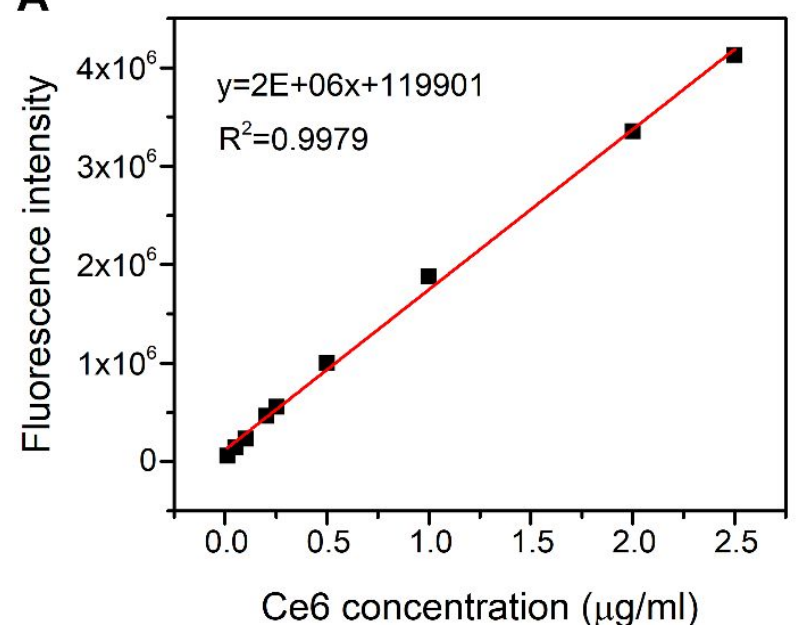

B

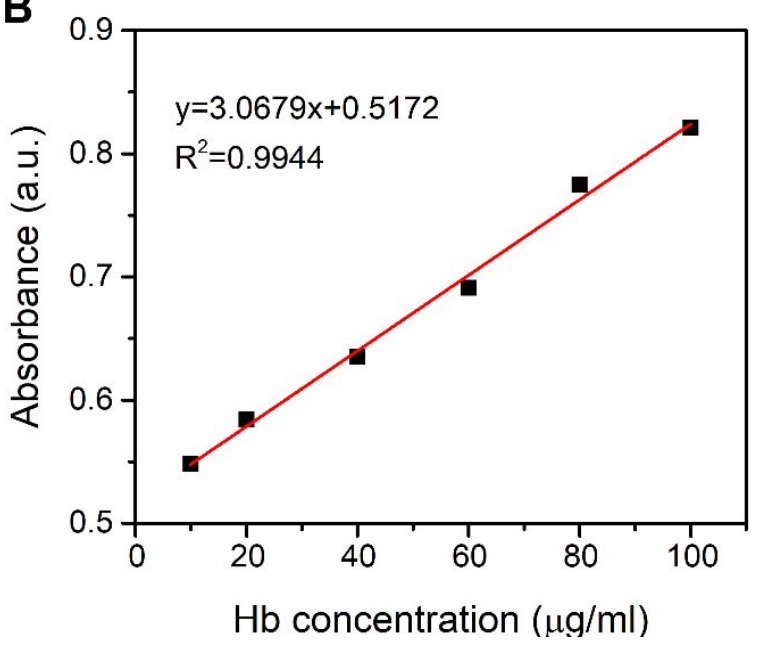

Figure S2. The standard curve for Ce6 (A) and $\mathrm{Hb}(\mathrm{B})$. 


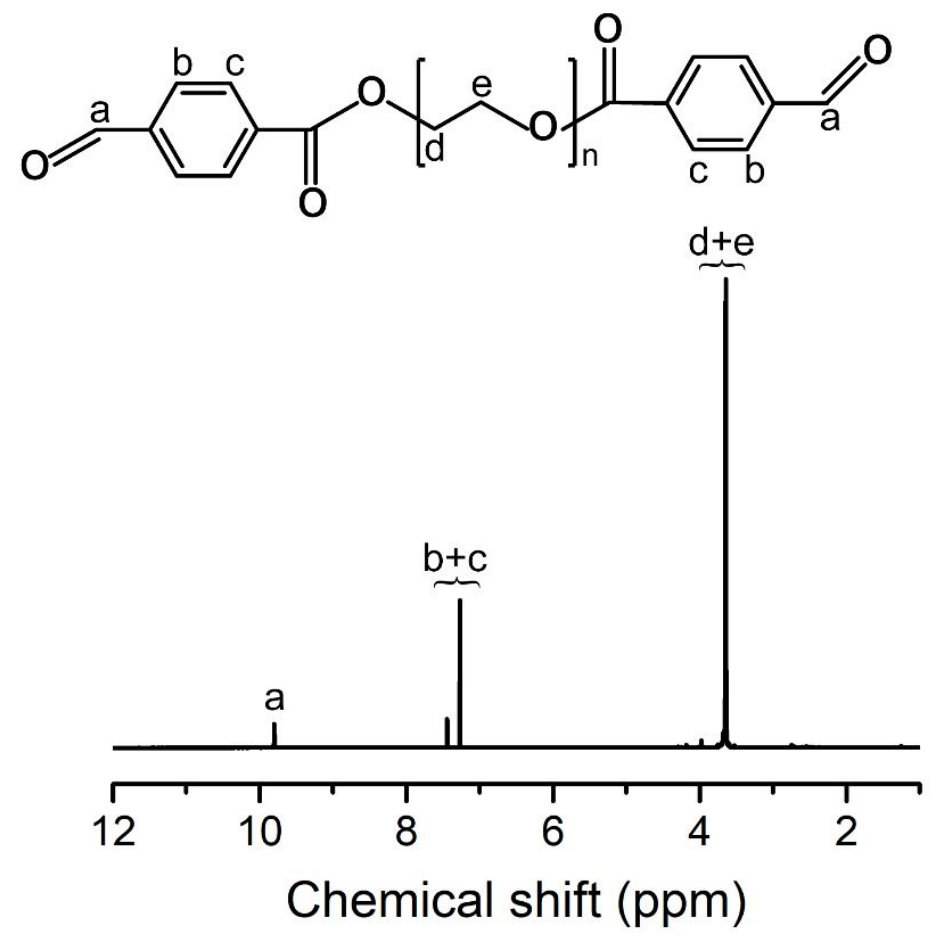

Figure S3. ${ }^{1} \mathrm{H}$ NMR spectrum of OHC-PEG-CHO. 


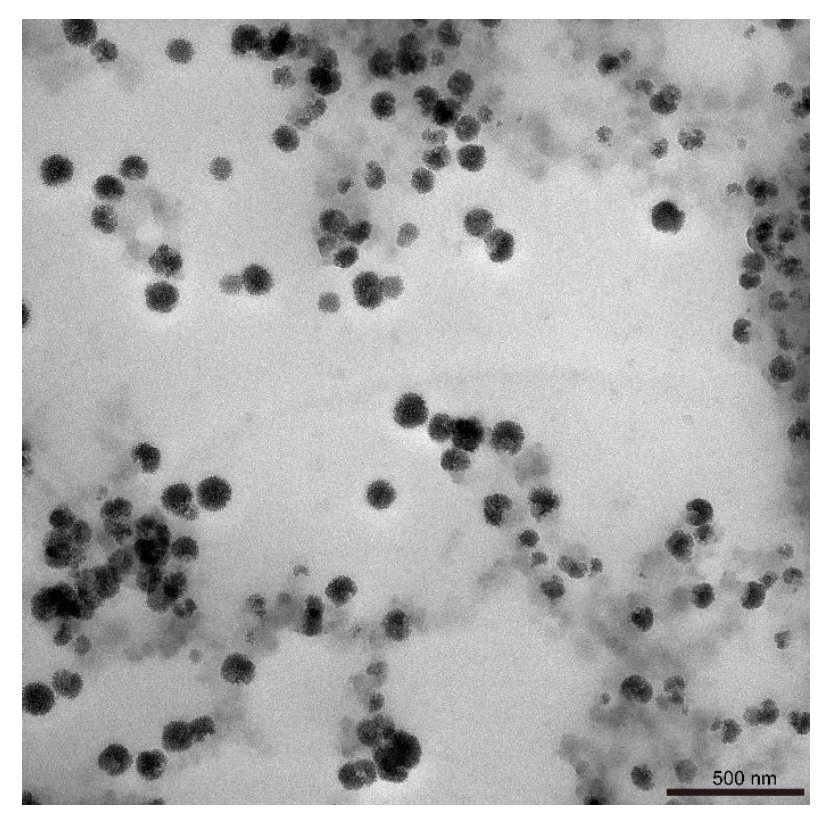

Figure S4. The TEM image of [PHC]PP NPs. 

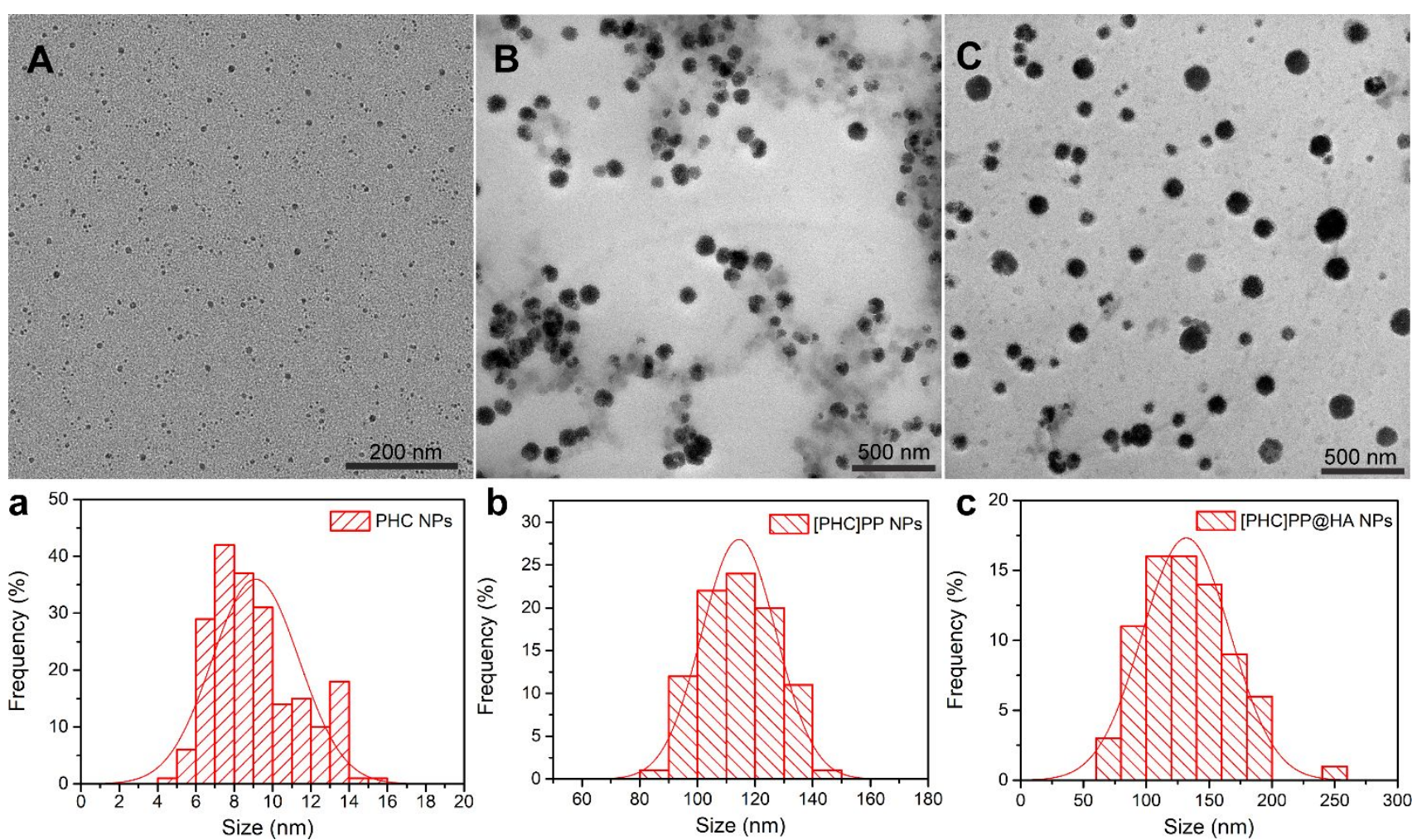

Figure S5. TEM images and size distribution of PHC NPs $(A, a),[P H C] P P ~ N P s ~(B, b)$ and [PHC]PP@HA NPs $(\mathrm{C}, \mathrm{c})$. 


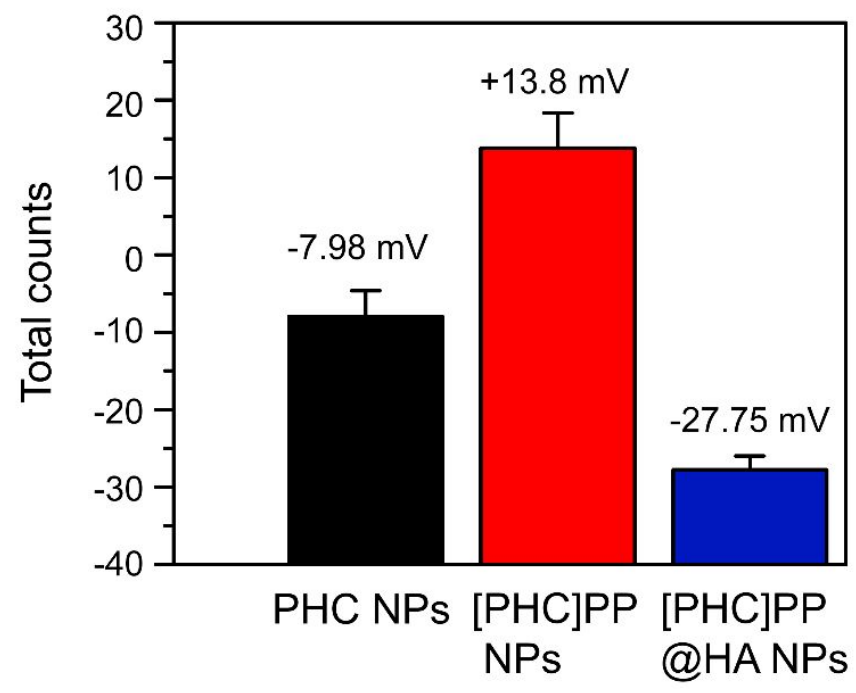

Figure S6. Zeta-potential of various samples.
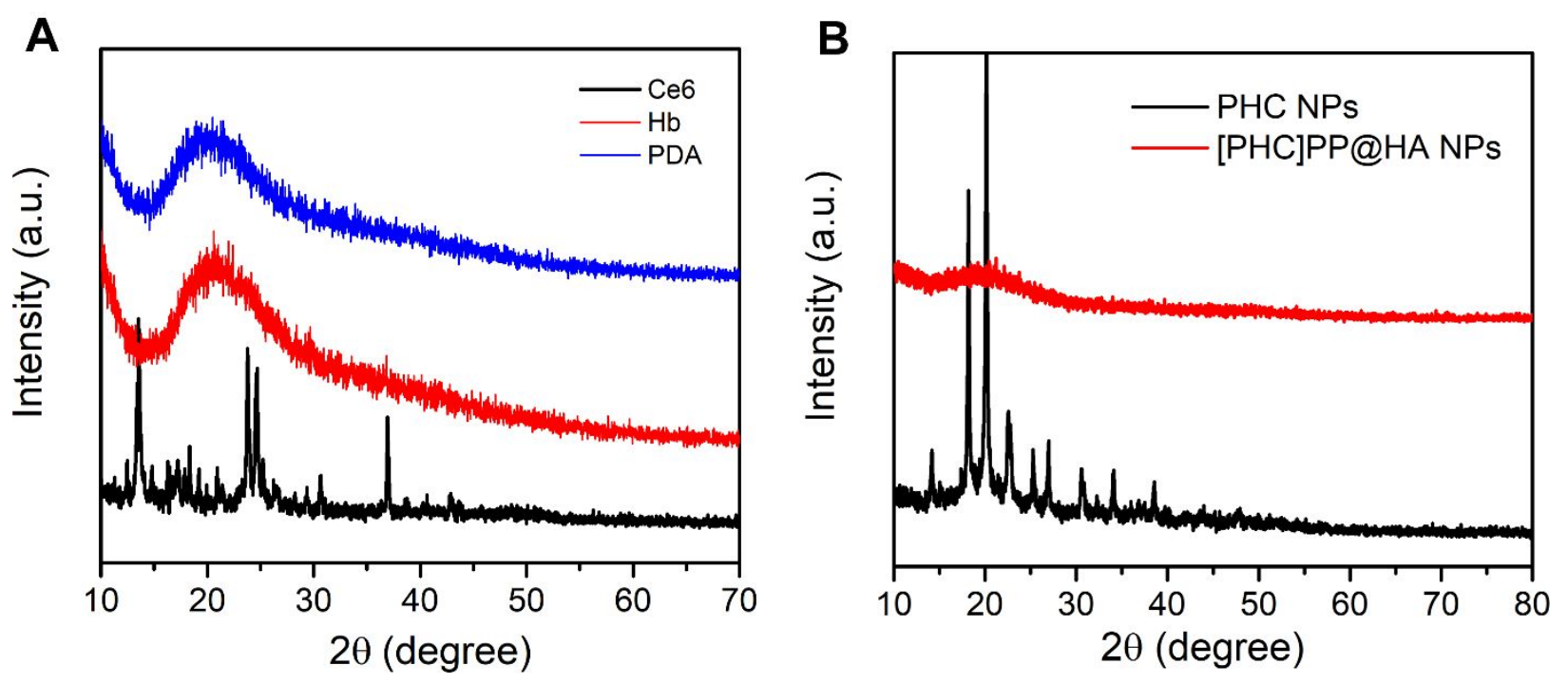

Figure S7. (A) The XRD patterns of PDA, Hb and Ce6. (B) The XRD patterns of PHC NPs and [PHC]PP@HA NPs. 

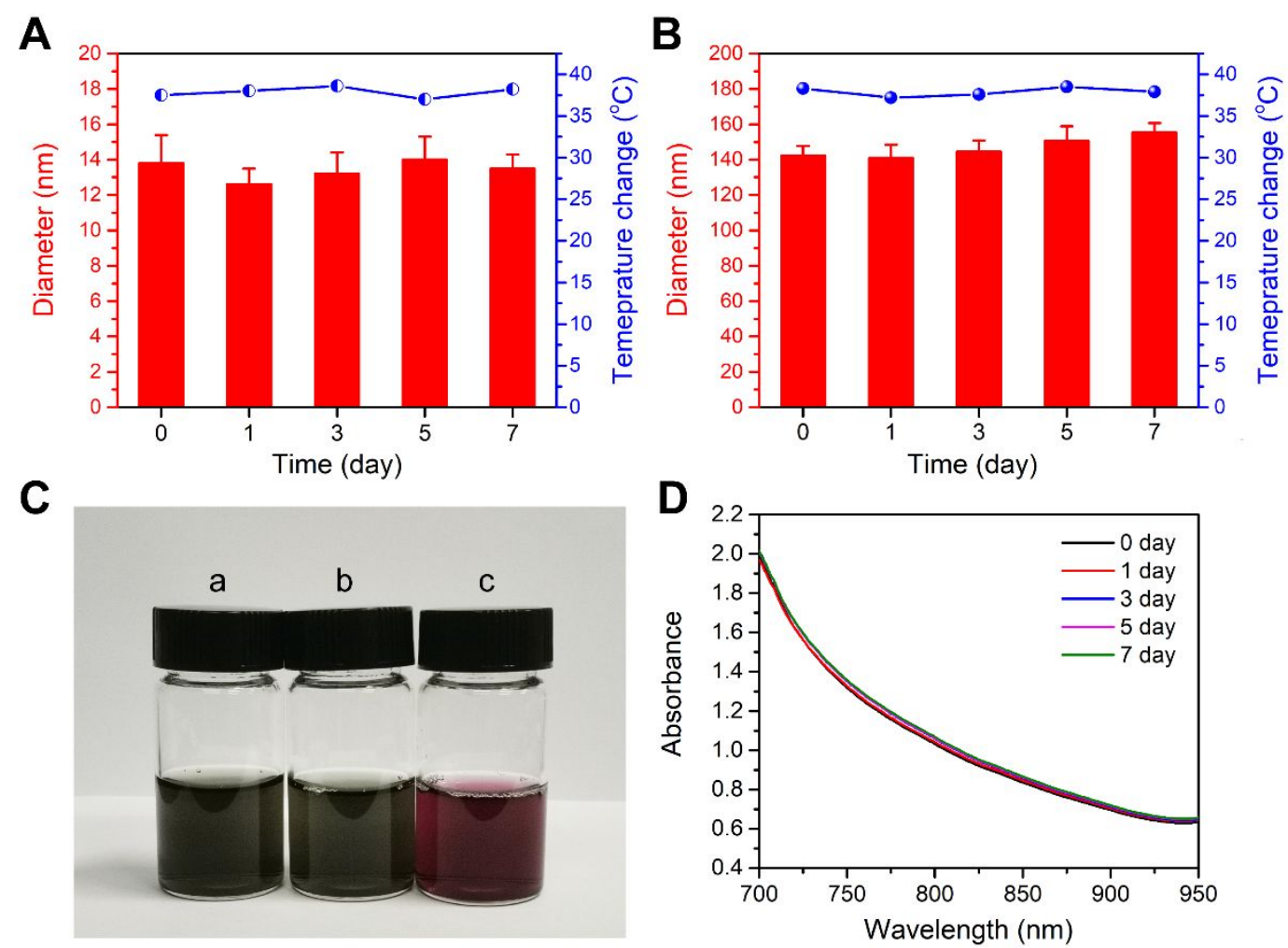

Figure S8. (A) Photothermal stability and colloid stability of PHC NPs in 7 days. (B) Photothermal stability and colloid stability of [PHC]PP@HA NPs in 7 days. (C) The stability of [PHC]PP@HA NPs dissolved in different media. (a) Pure water, (b) Glucose solution and (c) DMEM high-glucose cell medium containing 10\% fetal bovine serum. (D) The absorption of [PHC]PP@HA NPs in 1 week. 

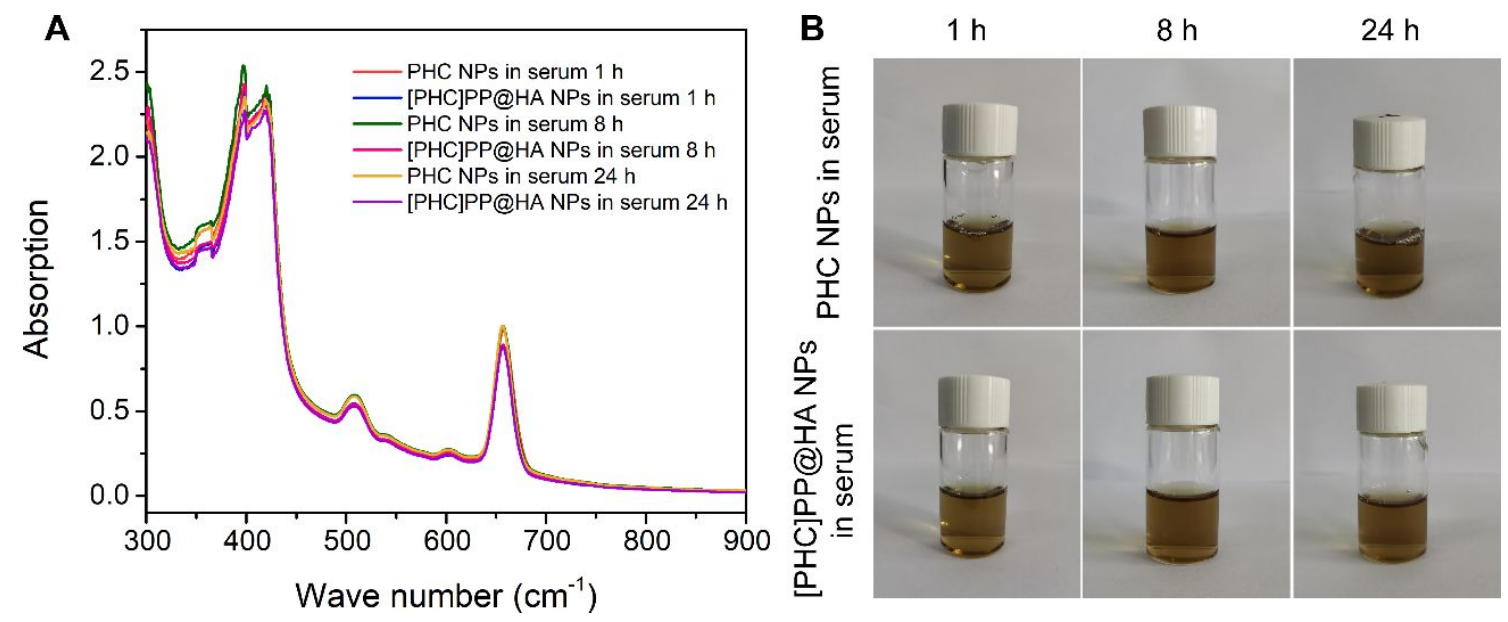

Figure S9. The colloidal stability of the [PHC]PP@HA NPs in serum for 24 hours. (A) The absorption of [PHC]PP@HA NPs in in serum for 24 hours. (B) Time-varying images of nanoparticles dispersed in serum for 24 hours. 

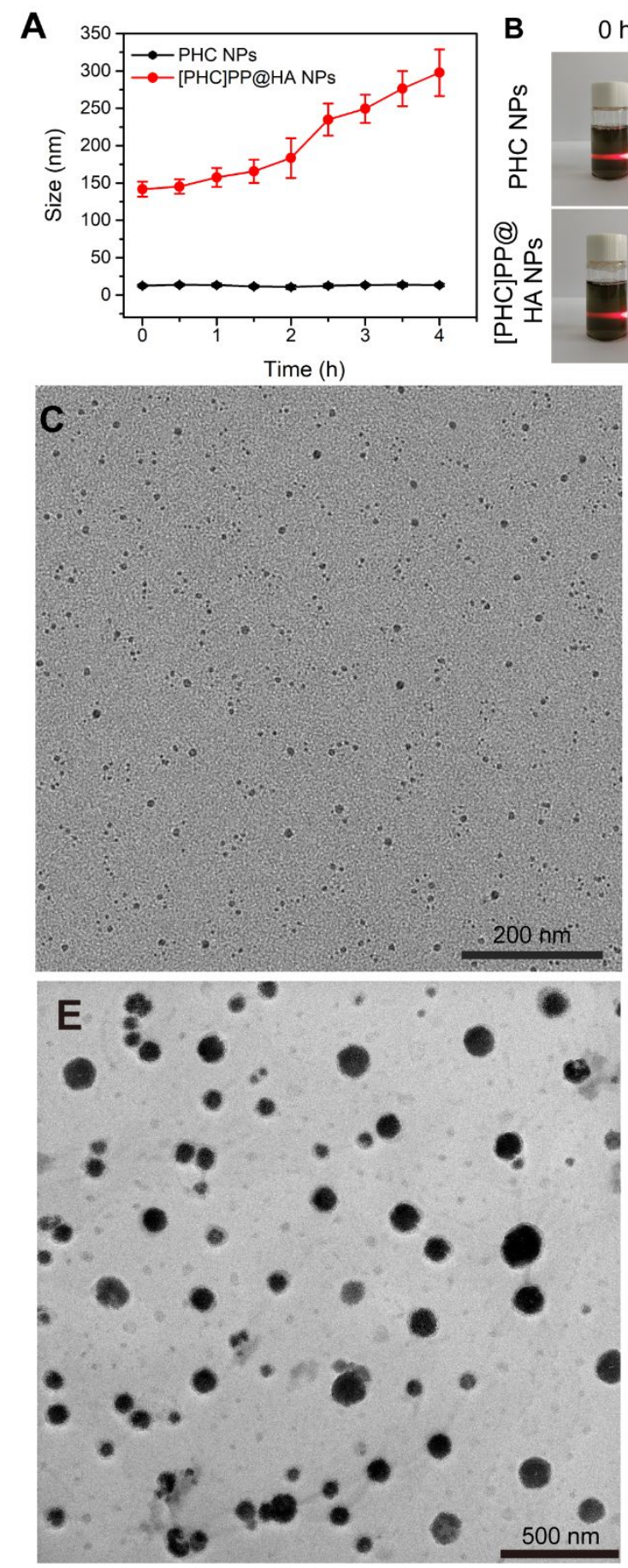
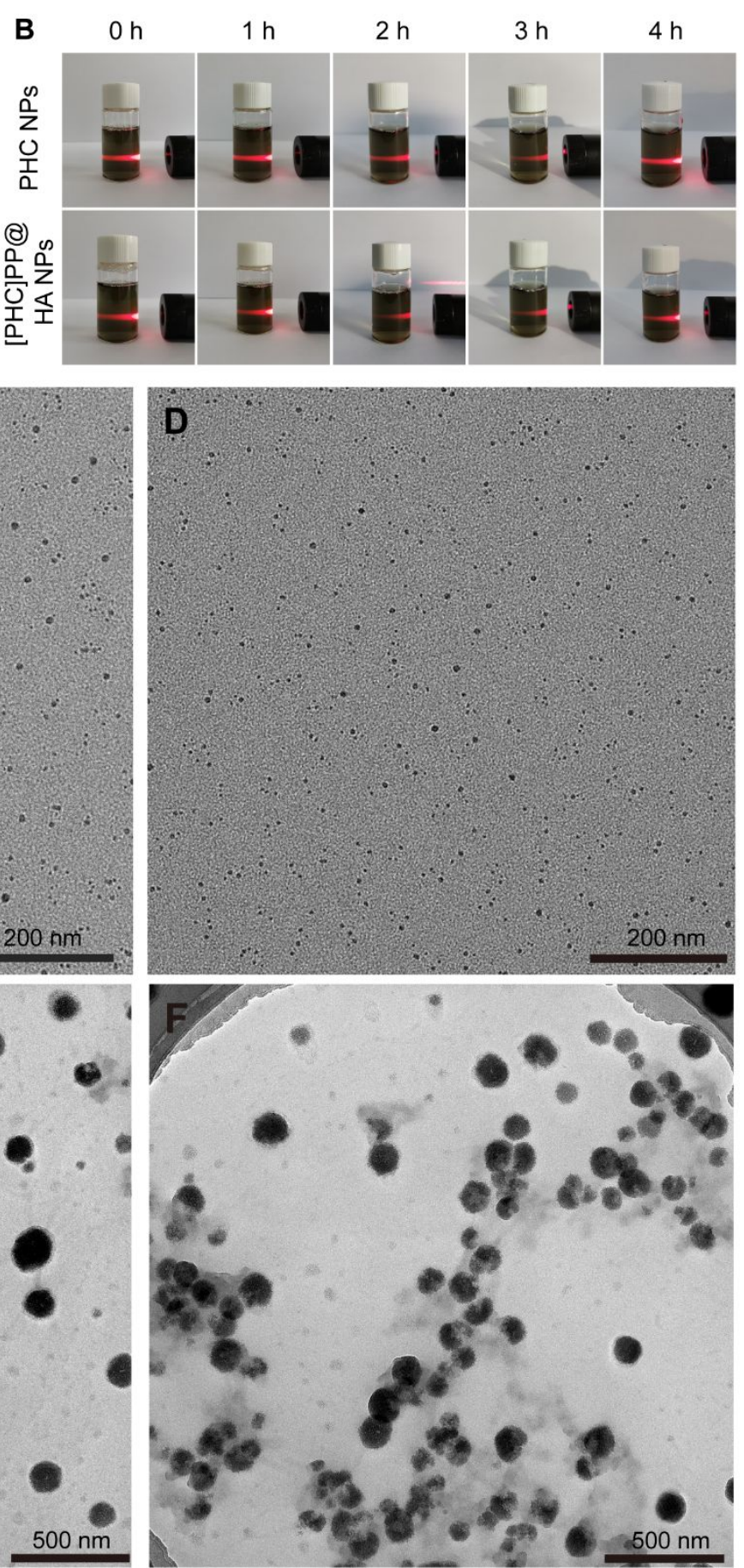

Figure S10. The thermal stability of PHC NPs and [PHC]PP@HA NPs. (A) The size change of PHC NPs and [PHC]PP@HA NPs at $55^{\circ} \mathrm{C}$ in 4 h. (B) The images of PHC NPs and [PHC]PP@HA NPs solution at $55^{\circ} \mathrm{C}$ in $4 \mathrm{~h}$. (C) TEM image of PHC NPs. (D) TEM image of PHC NPs irradiated with 808 nm laser for 10 min. (E) TEM image of [PHC]PP@HA NPs. (F) TEM image of [PHC]PP@HA NPs irradiated with $808 \mathrm{~nm}$ laser for $10 \mathrm{~min}$. 


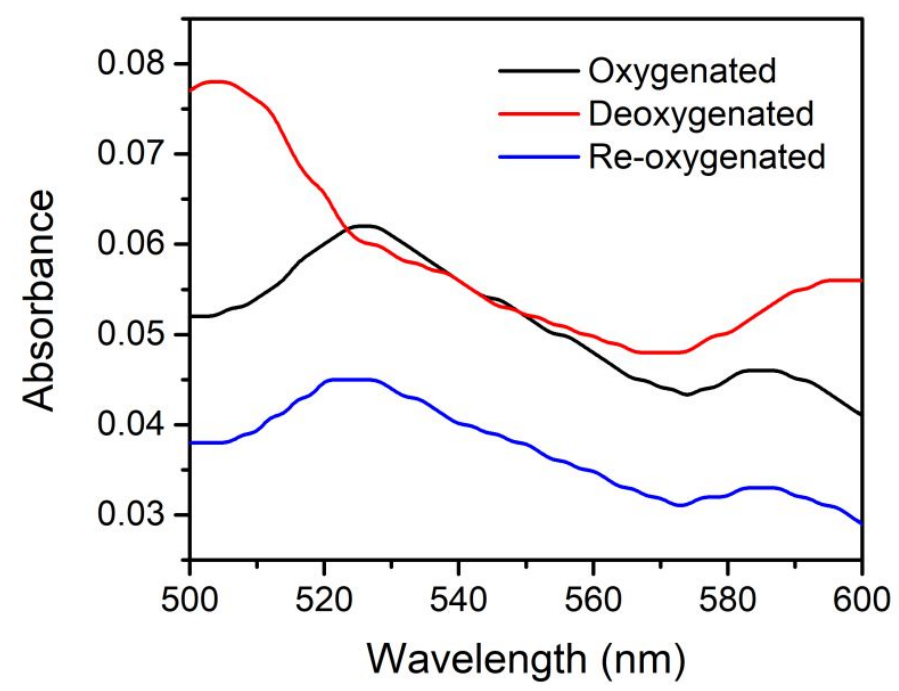

Figure S11. UV-vis absorption spectra of oxygenated, deoxygenated and reoxygenated [PHC]PP@HA NPs in pH 7.4 PBS $\left(25^{\circ} \mathrm{C}\right)$.

The absorption peak of $\mathrm{Hb}$ at 500-600 $\mathrm{nm}$ varies with the ligand state of the heme and oxygenation of $\mathrm{Hb}$. The oxygenated [PHC]PP@HA NPs had obvious absorption peak of $\mathrm{Hb}$ at about $530 \mathrm{~nm}$, while the characteristic peak of deoxygenated [PHC]PP@HA NPs at 530 nm disappeared. Subsequently, the absorption peak of $\mathrm{Hb}$ at $530 \mathrm{~nm}$ was reappeared after the sample was re-oxygenated, indicating that the $\mathrm{Hb}$ in $[\mathrm{PHC}] \mathrm{PP} @ \mathrm{HA}$ NPs has complete structure and the ability to carry and release oxygen. 


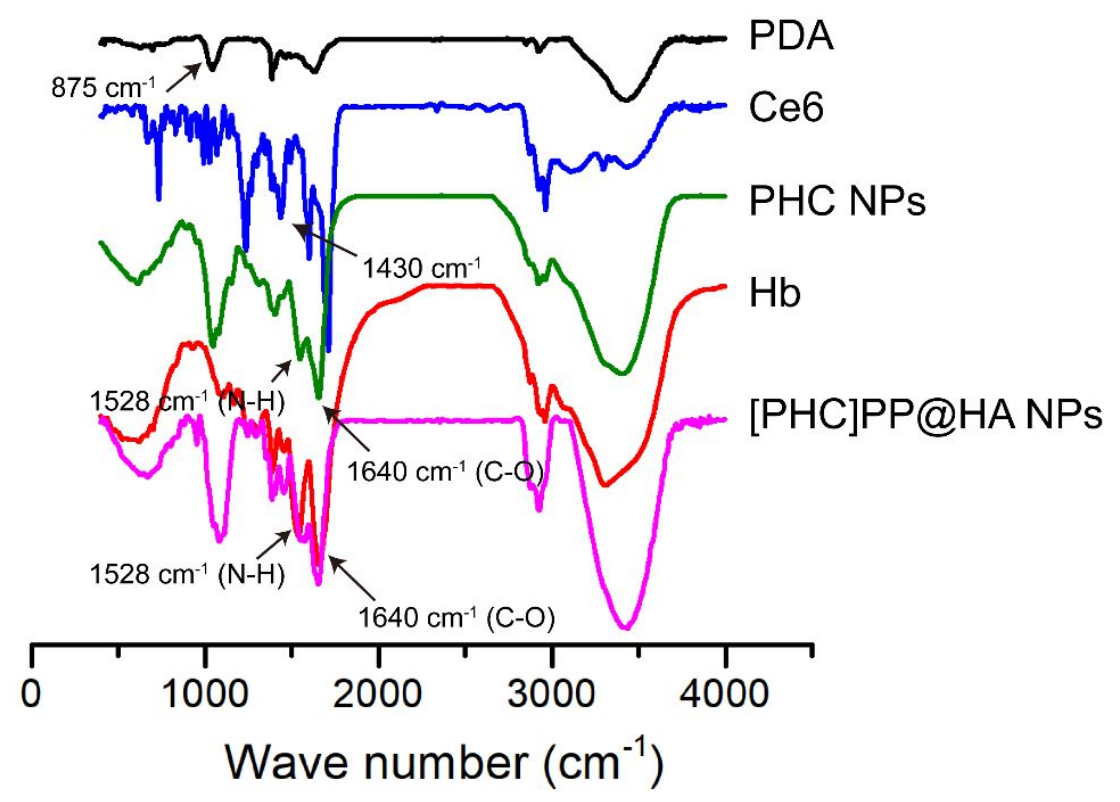

Figure S12. FT-IR spectra of various samples.

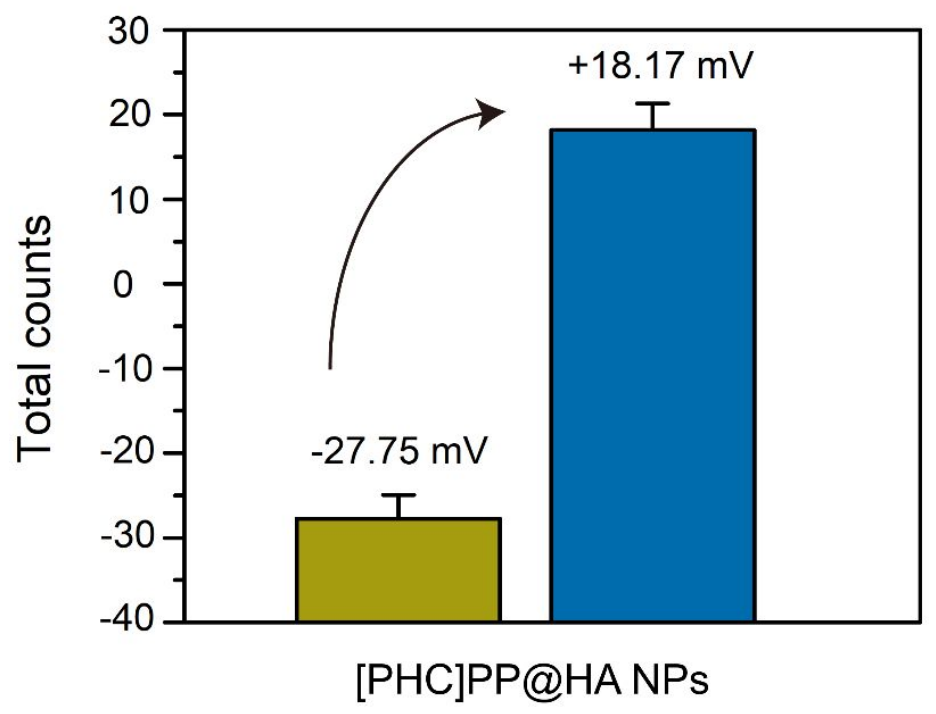

Figure S13. Characterization of Zeta-potential variation of [PHC]PP@HA NPs at pH 6.8 and pH 7.4 by dynamic light scattering (DLS). 


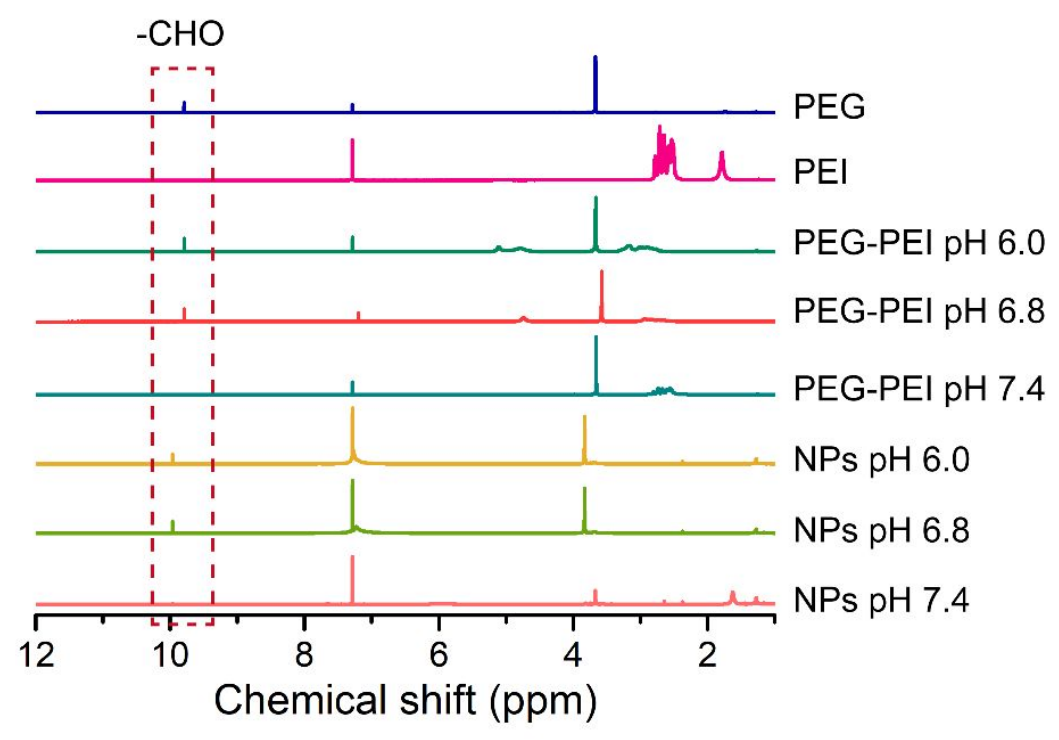

Figure S14. ${ }^{1} \mathrm{H}$ NMR spectra of PEI, OHC-PEG-CHO and the $\mathrm{pH}$-sensitivity of the benzoic-imine bonds generated between PEI and OHC-PEG-CHO. In neutral solution (pH 7.4), the aldehyde group of OHC-PEG-CHO could react with the amino groups of PEI to form benzoic-imine bonds. In the ${ }^{1} \mathrm{H}$ NMR spectra of PEG-PEI and [PHC]PP@HA NPs, the peak of aldehyde groups (at 9.7 ppm) completely disappeared in $\mathrm{pH} 7.4$ which meant that all of the aldehyde groups reacted with PEI to form benzoic-imine bonds. However, the acidic $\mathrm{pH}(\mathrm{pH} 6.8$ and 6.0) could rapidly cleave the benzoic-imine bonds and restored the signal of aldehyde groups in ${ }^{1} \mathrm{H}$ NMR spectrum. This result sufficiently demonstrated the sensitive pH-response of the [PHC]PP@HA NPs. 


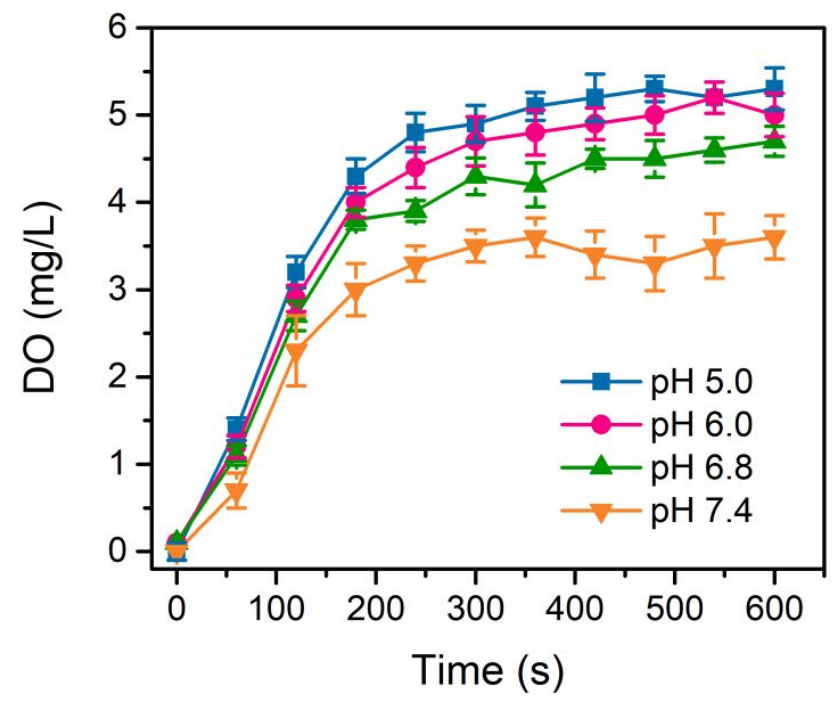

Figure S15. The oxygen release behavior of [PHC]PP@HA NPs in different pH with the concentration of $5 \mathrm{mg} \mathrm{mL}^{-1} \mathrm{Hb}$.
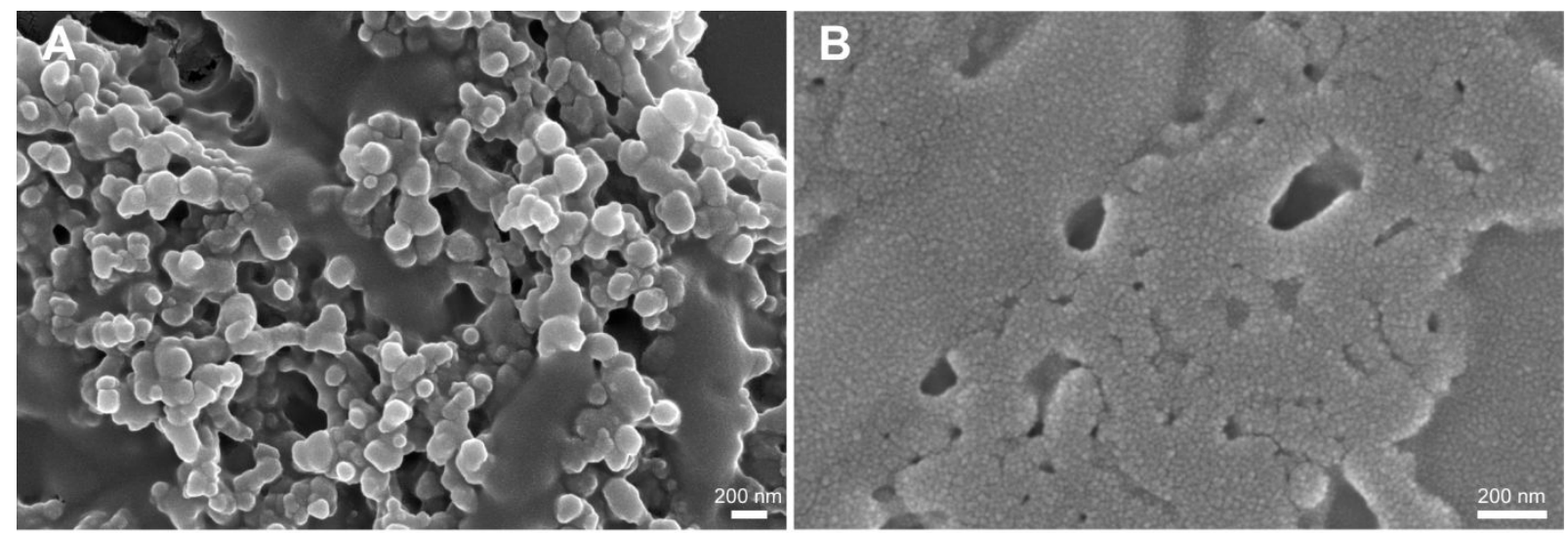

Figure S16. The SEM images of [PHC]PP@HA NPs after soaked in buffers with different pH 7.4 (A) and $\mathrm{pH} 6.8(\mathrm{~B})$. 

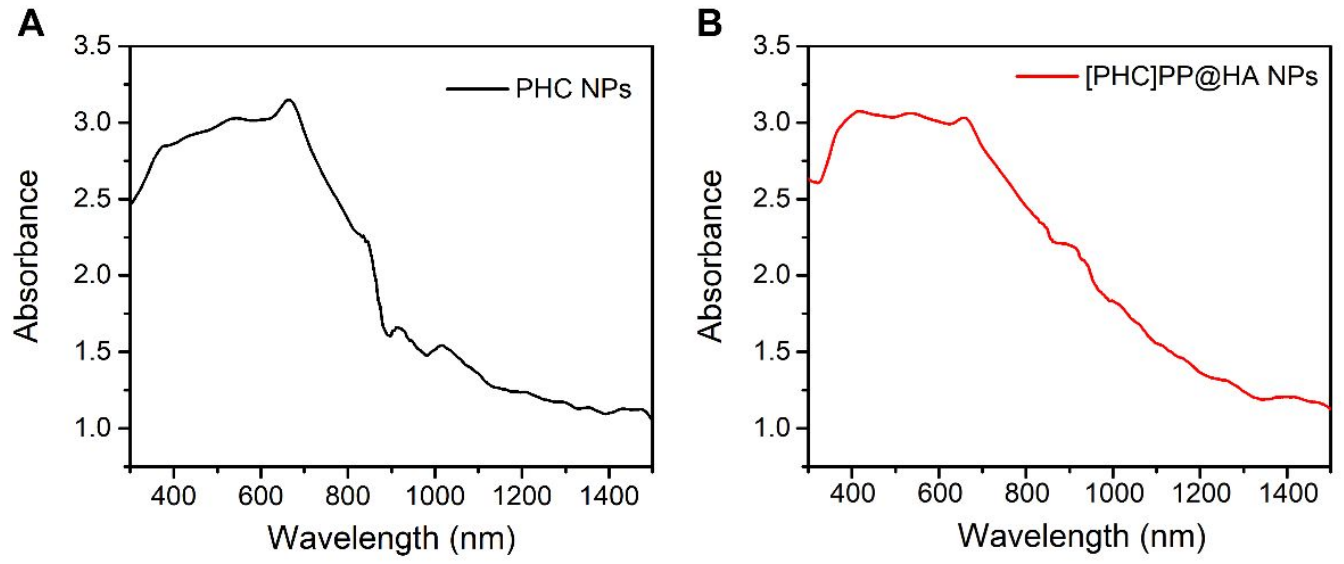

Figure S17. Powder absorbance of PHC NPs (A) and [PHC]PP@HA NPs (B). 


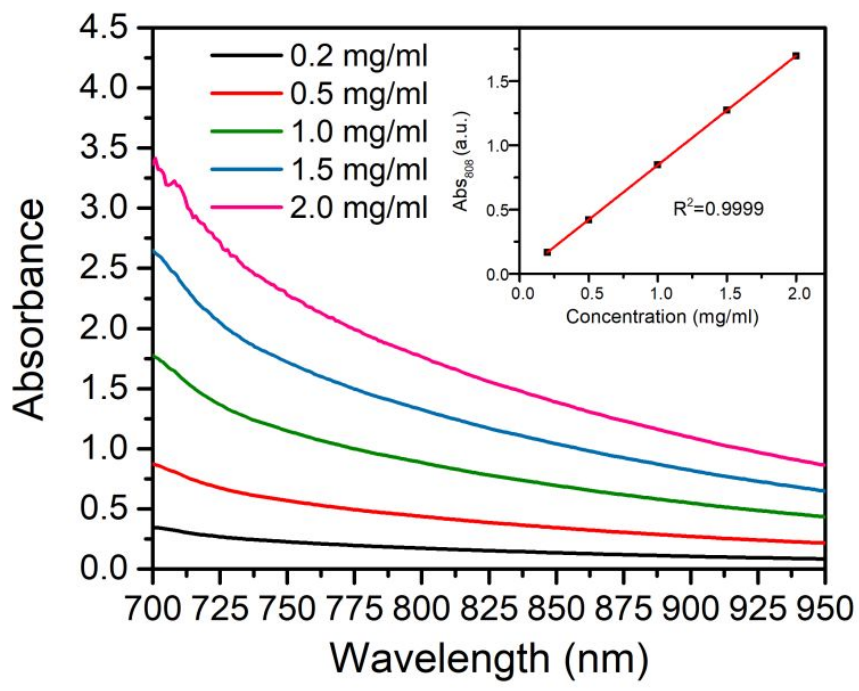

Figure S18. The absorption spectra of [PHC]PP@HA NPs with different concentrations.

A

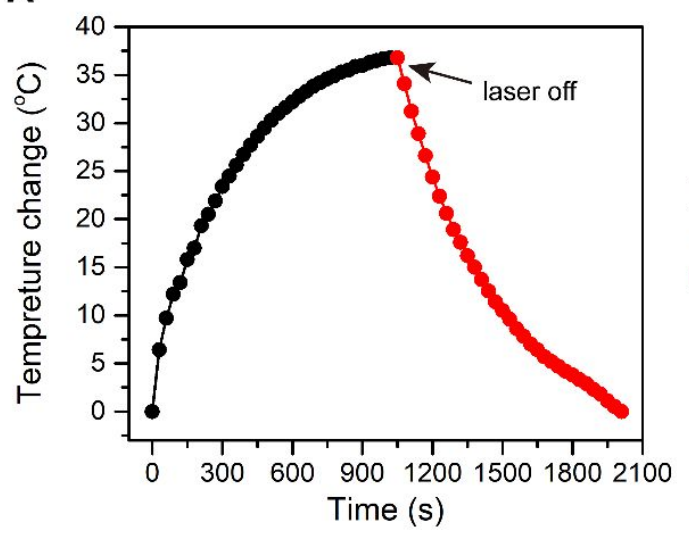

B

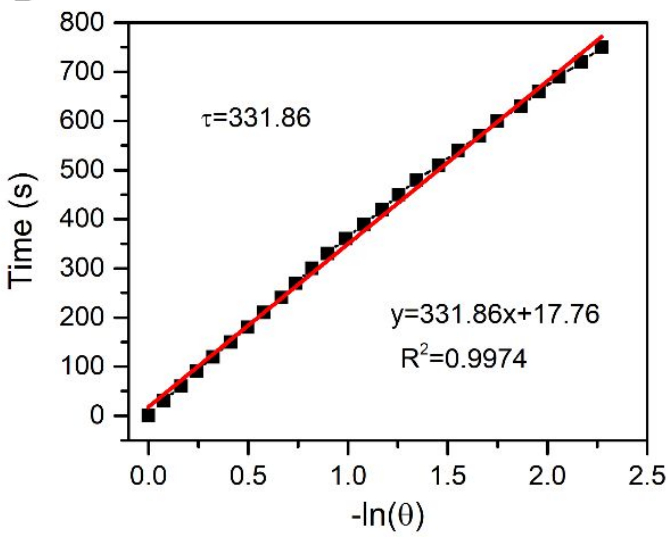

Figure S19. (A) The photothermal profile of [PHC]PP@HA NPs, and then switched off the laser and naturally cooled the solution $\left(808 \mathrm{~nm}, 1 \mathrm{~W} \mathrm{~cm}^{-2}\right)$. (B) System time constant for heat transfer from the nanosystem was calculated to be $331.86 \mathrm{~s}$ by applying the linear time data from the cooling period in A. 


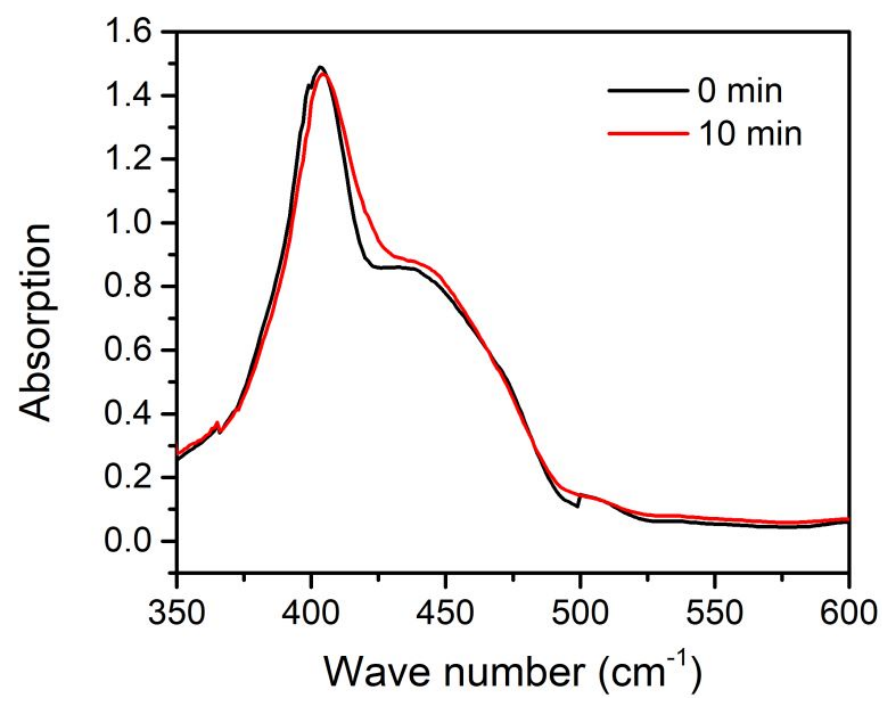

Figure S20. Changes of absorption spectra of RNO by ROS generation upon $808 \mathrm{~nm}$ laser $\left(1 \mathrm{~W} \mathrm{~cm}^{-2}\right)$ irradiation in [PHC]PP@HA NPs solution.
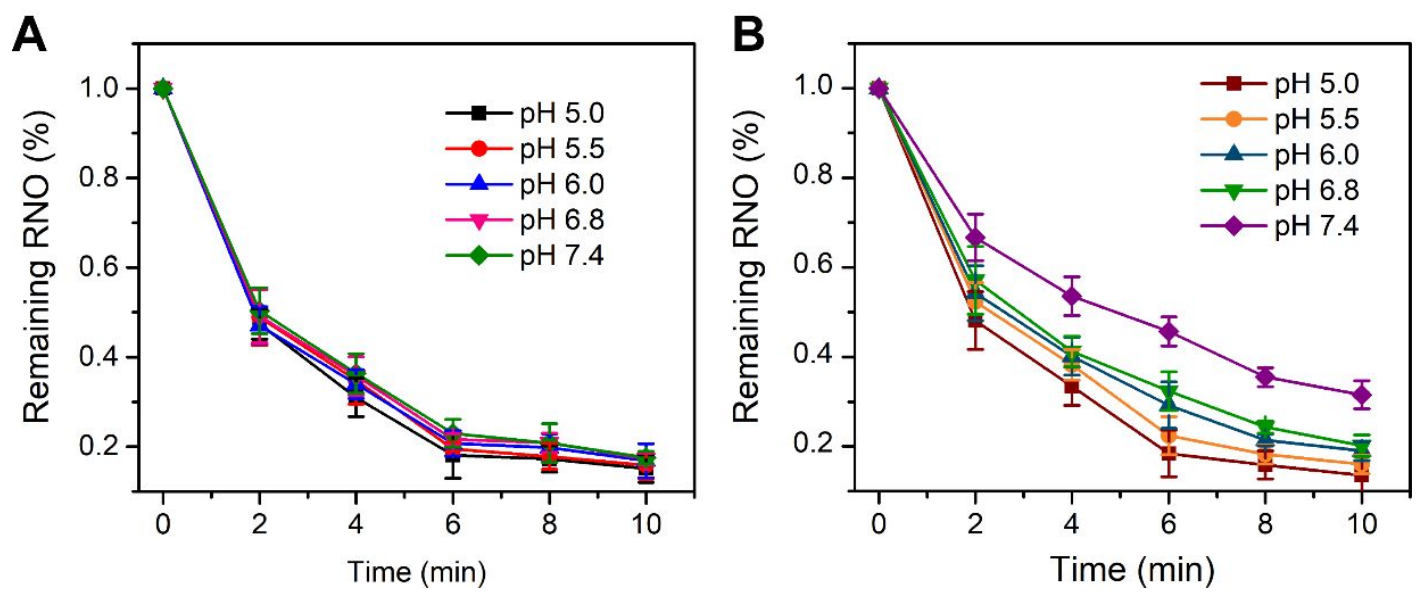

Figure S21. Normalized absorbance of RNO at $440 \mathrm{~nm}$ during ROS detection in using RNO assay in PHC NPs (A) and [PHC]PP@HA NPs (B) solutions with different pH. 

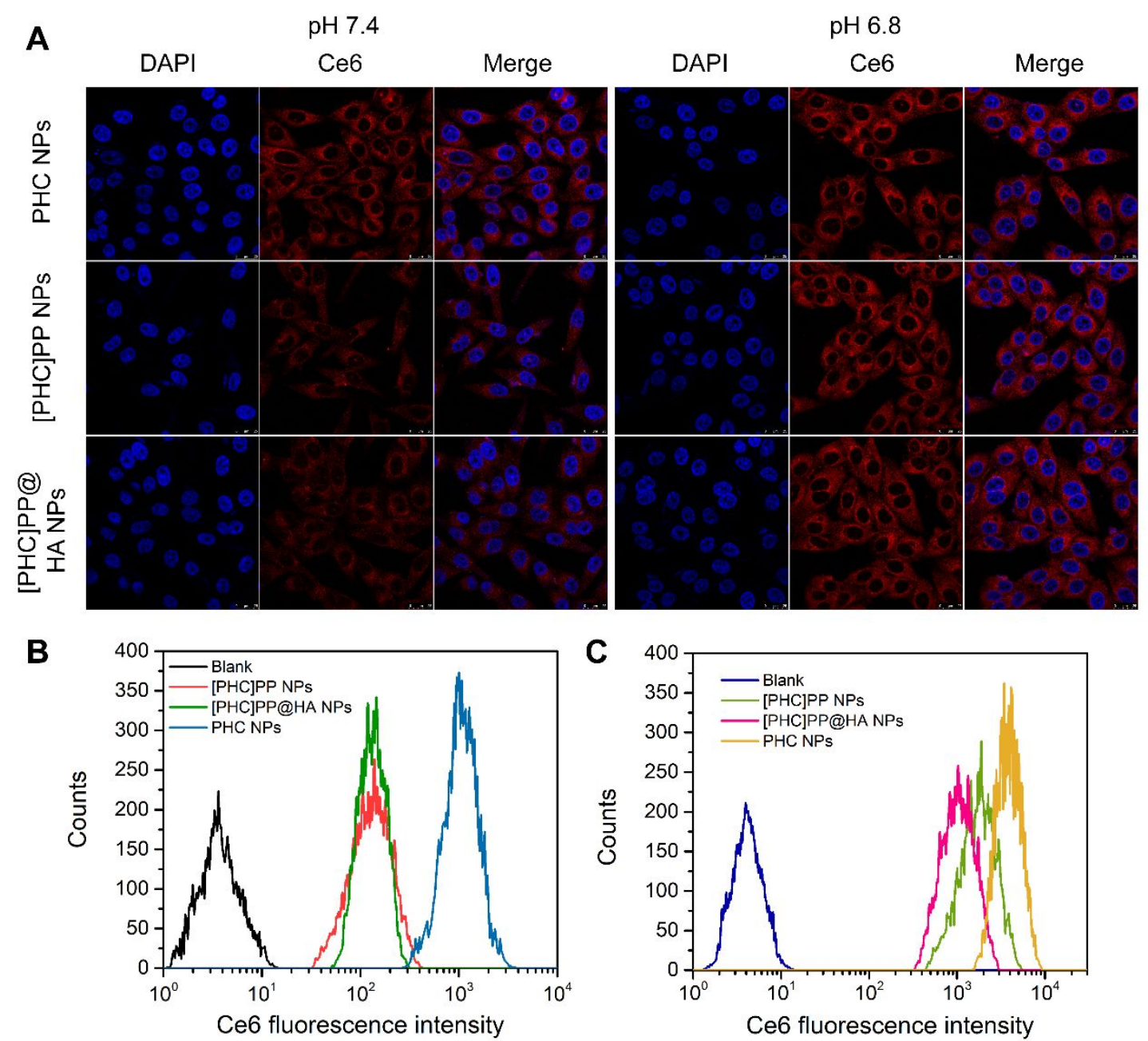

Figure S22. (A) CLSM images of PC-3 cells treated with PHC NPs, [PHC]PP NPs and [PHC]PP@HA NPs at different $\mathrm{pH}$ (6.8 and 7.4) for $4 \mathrm{~h}$. The scale bar is $25 \mu \mathrm{m}$. (B) Flow cytometry analysis of cells at $\mathrm{pH}$ 7.4. (C) Flow cytometry analysis of cells at $\mathrm{pH} 6.8$. 


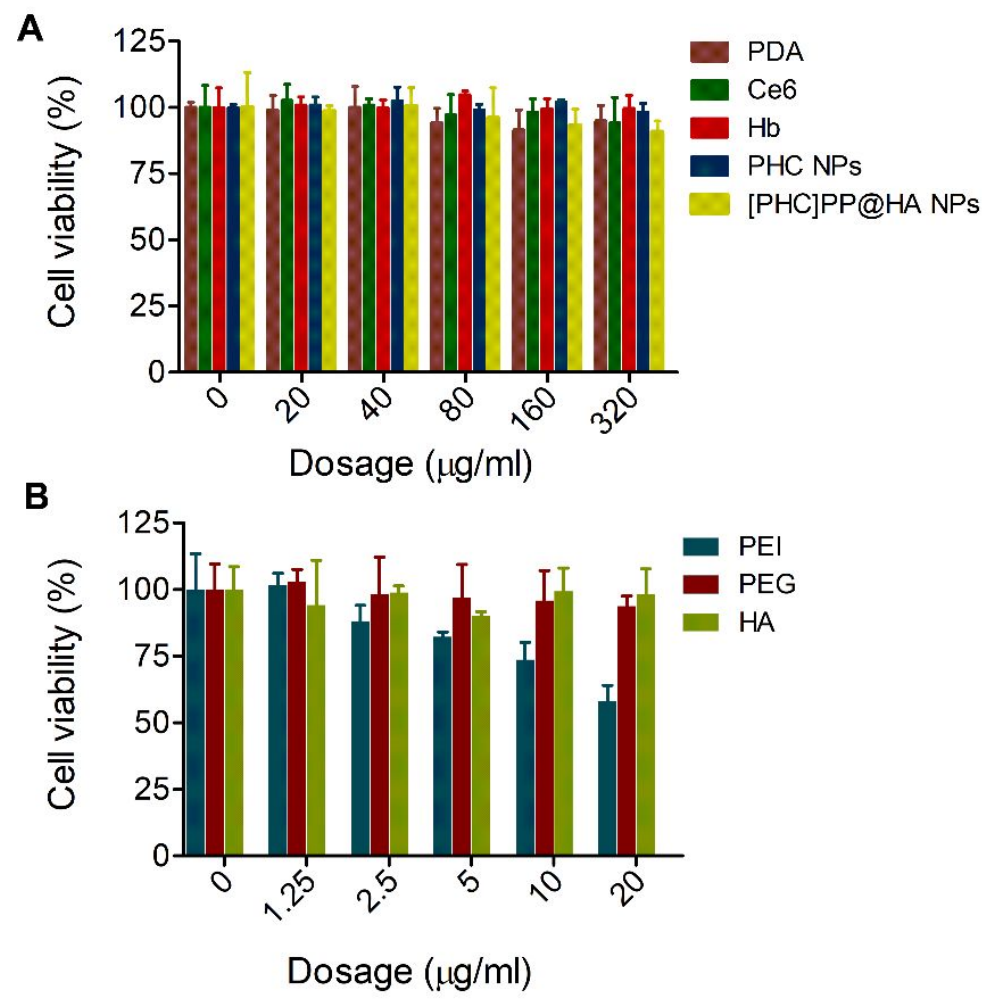

Figure S23. Relative cell viability after treated with various concentrations of samples for $48 \mathrm{~h}$. 

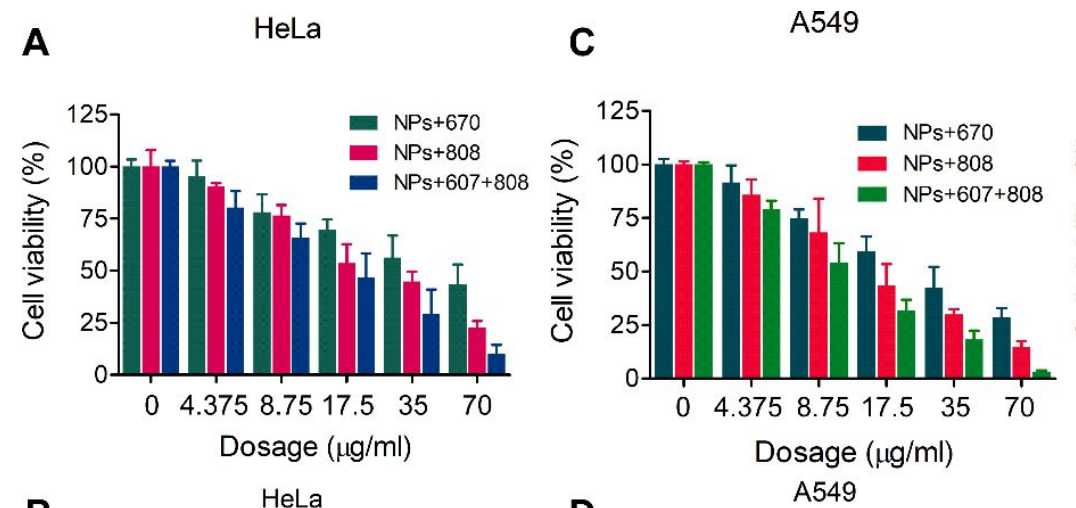

E PANC-1

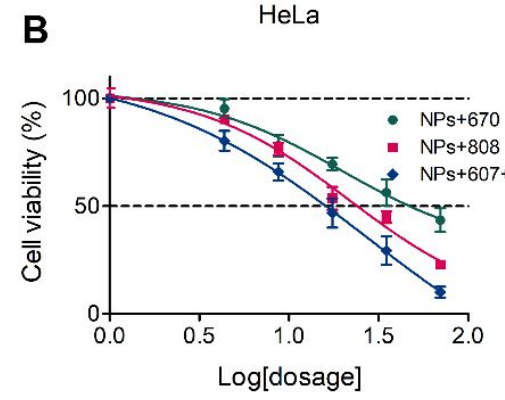

D

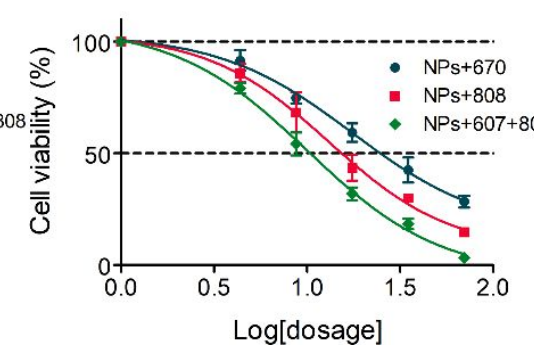

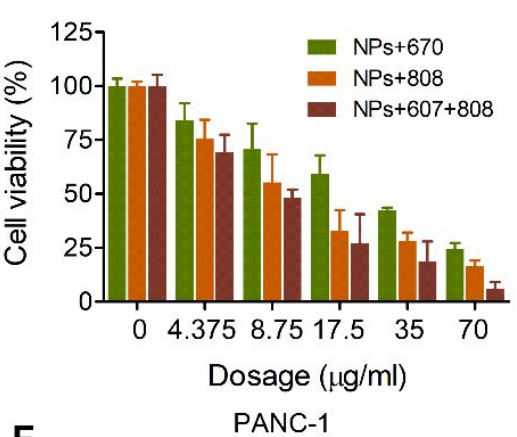

F

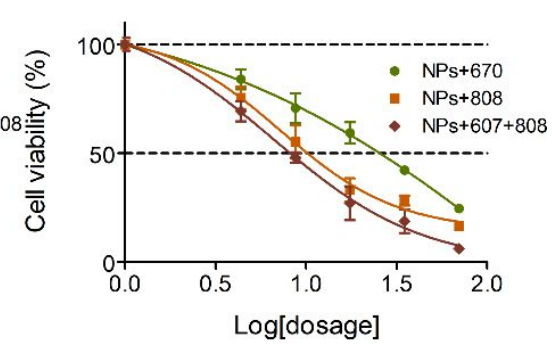

Figure S24. Viability of HeLa, A549 and PANC-1 cells treated with [PHC]PP@HA NPs under different laser. 

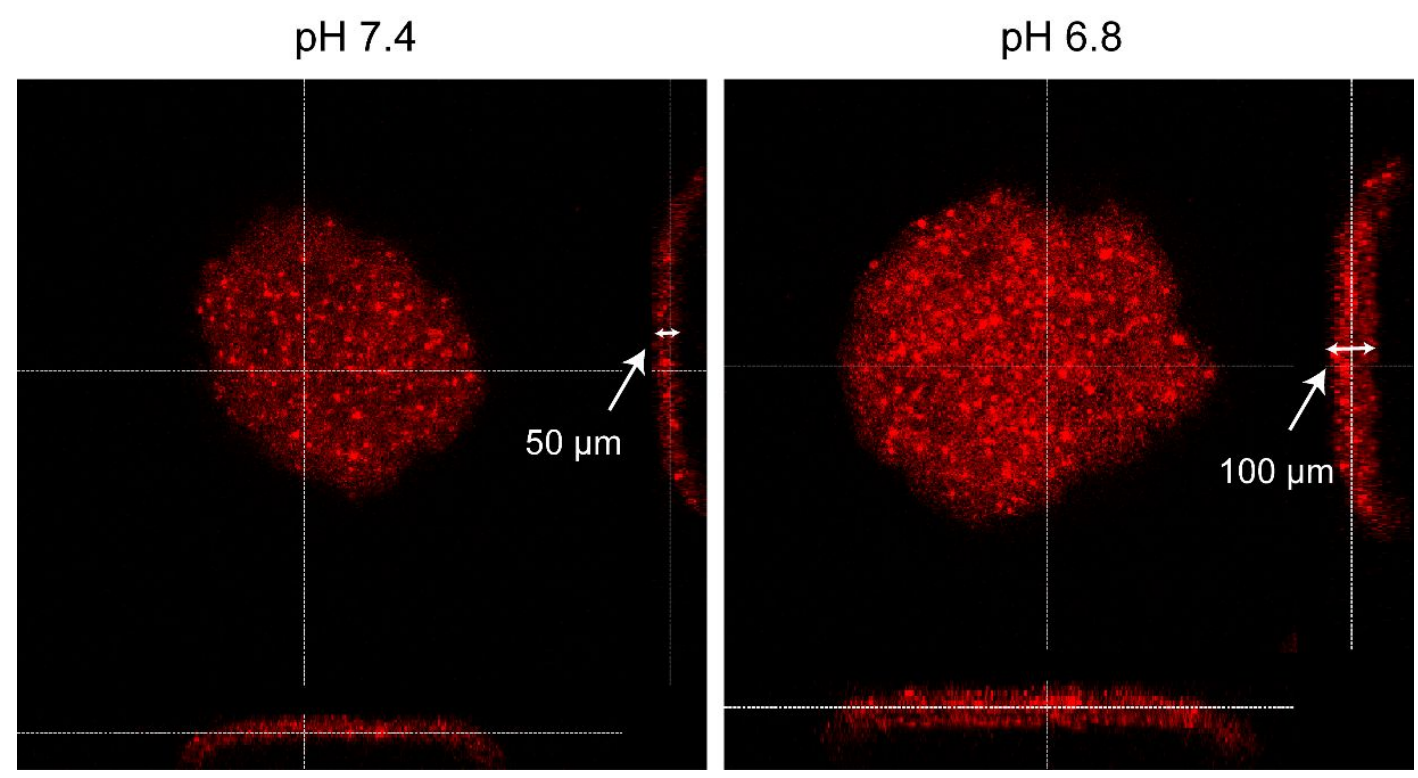

Figure S25. Confocal microscopy images of A549 spheroids after the treatment of [PHC]PP@HA NPs at pH 6.8 and pH 7.4.

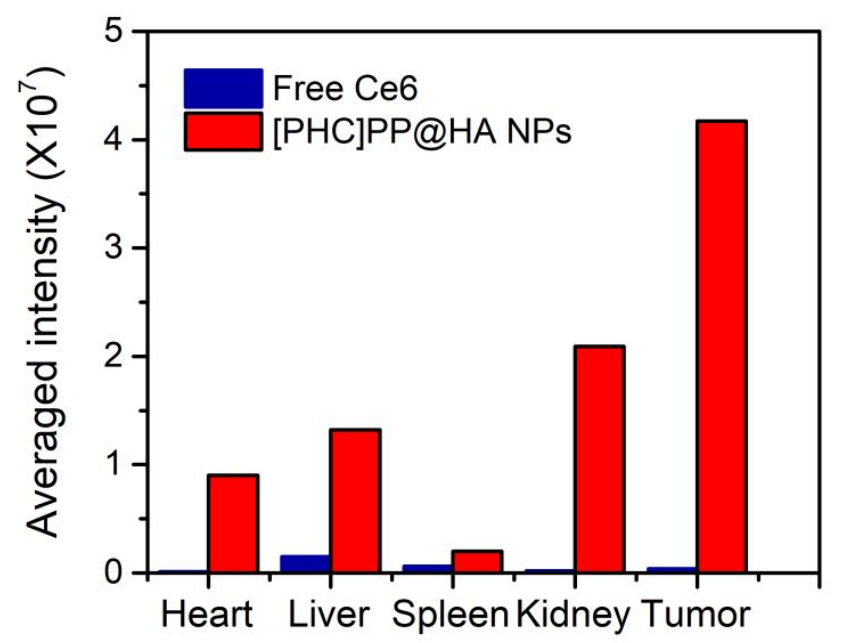

Figure S26. The fluorescence intensity of tumor and major organs from PC-3 tumor-bearing mice at $48 \mathrm{~h}$ postinjection. 


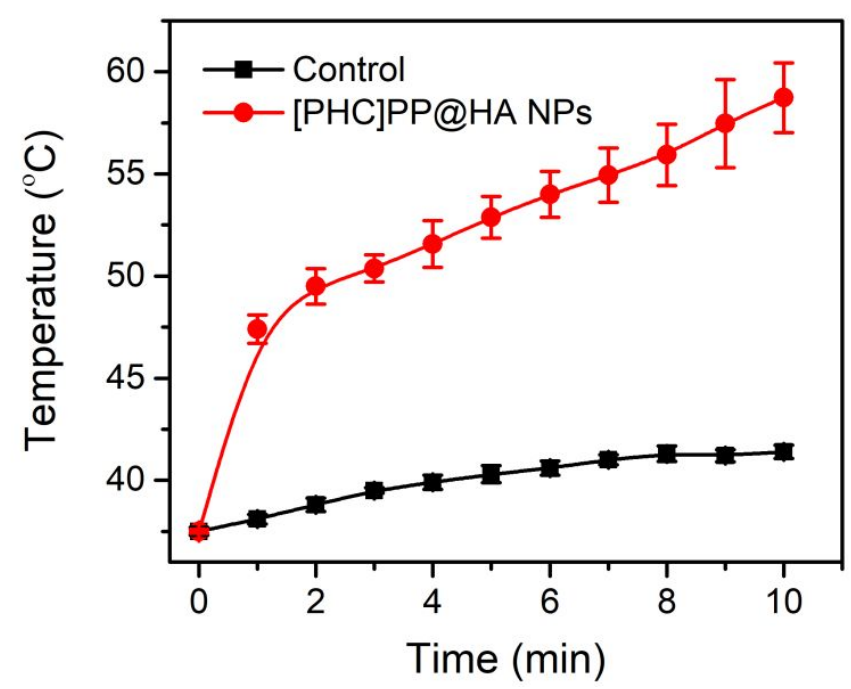

Figure S27. In vivo temperatures changes in tumor area of mice injected intravenous with [PHC]PP@HA NPs or PBS upon 10 min laser irradiation $\left(808 \mathrm{~nm}, 1 \mathrm{~W} \mathrm{~cm}\right.$-2$^{-2}$. 


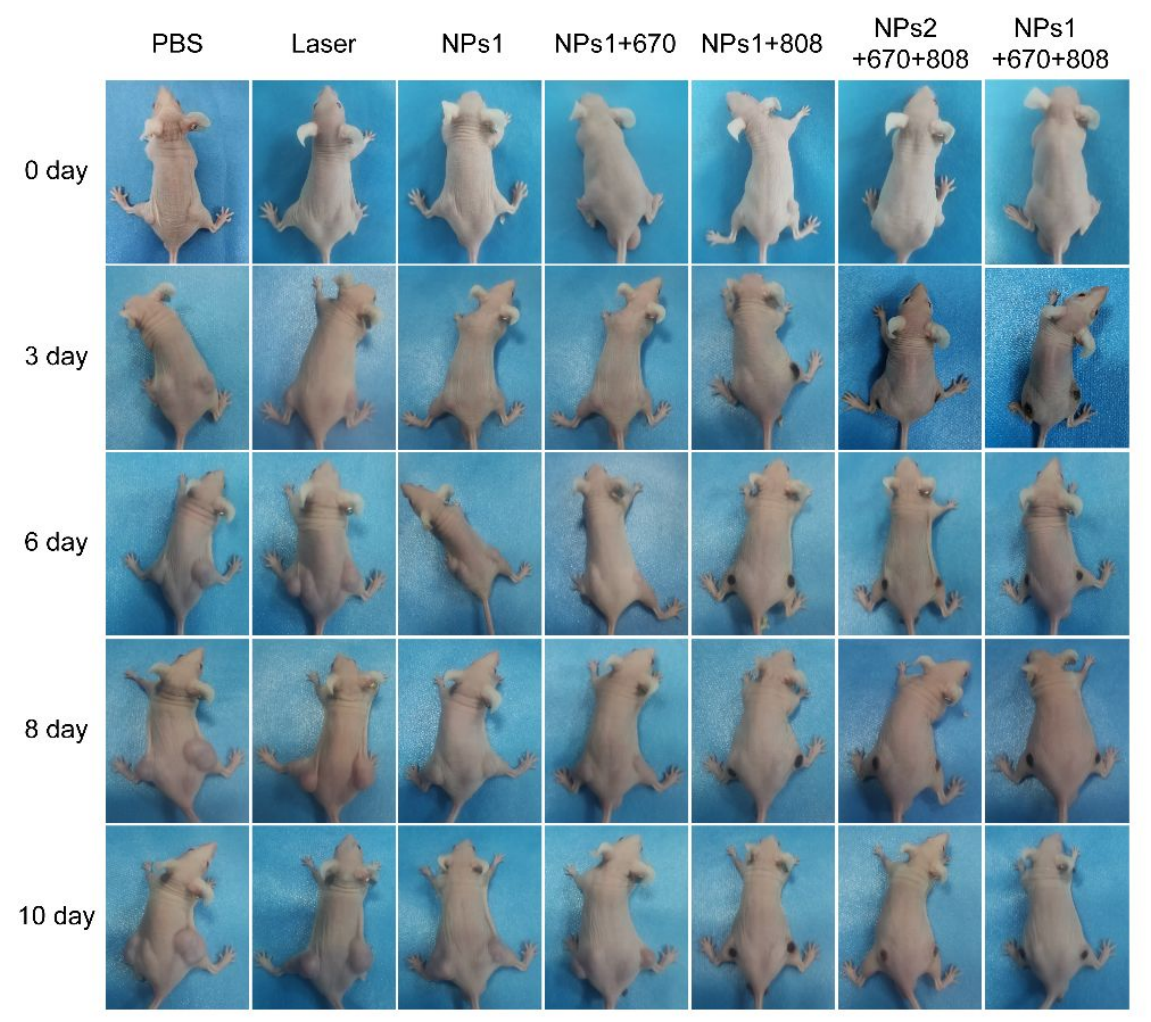

Figure S28. Photographs of mice after different treatments at different days. NPs 1 and NPs2 represent [PHC]PP@HA NPs and [PC]PP@HA NPs, respectively. 


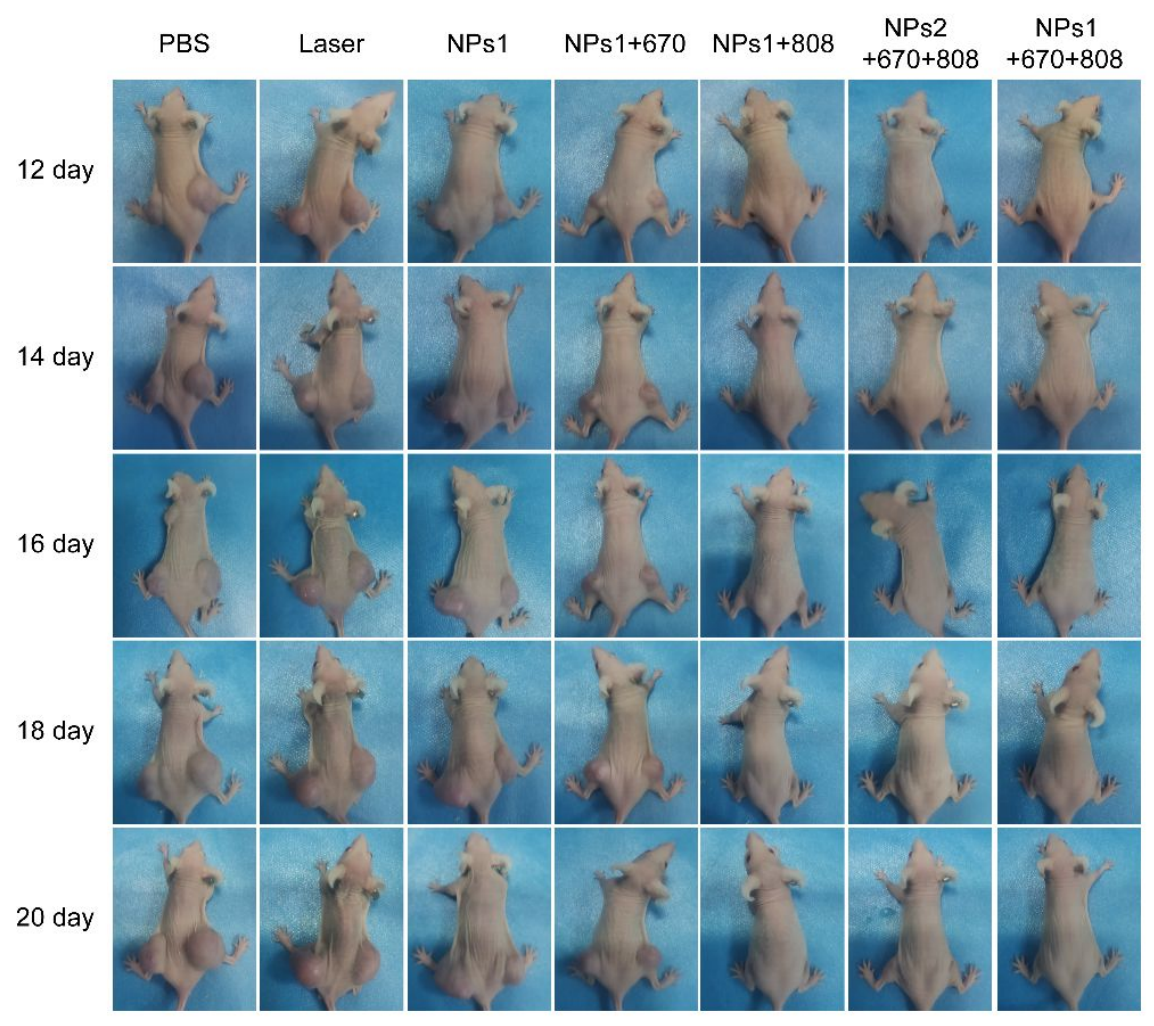

Figure S29. Photographs of mice after different treatments at different days. NPs 1 and NPs2 represent [PHC]PP@HA NPs and [PC]PP@HA NPs, respectively. 

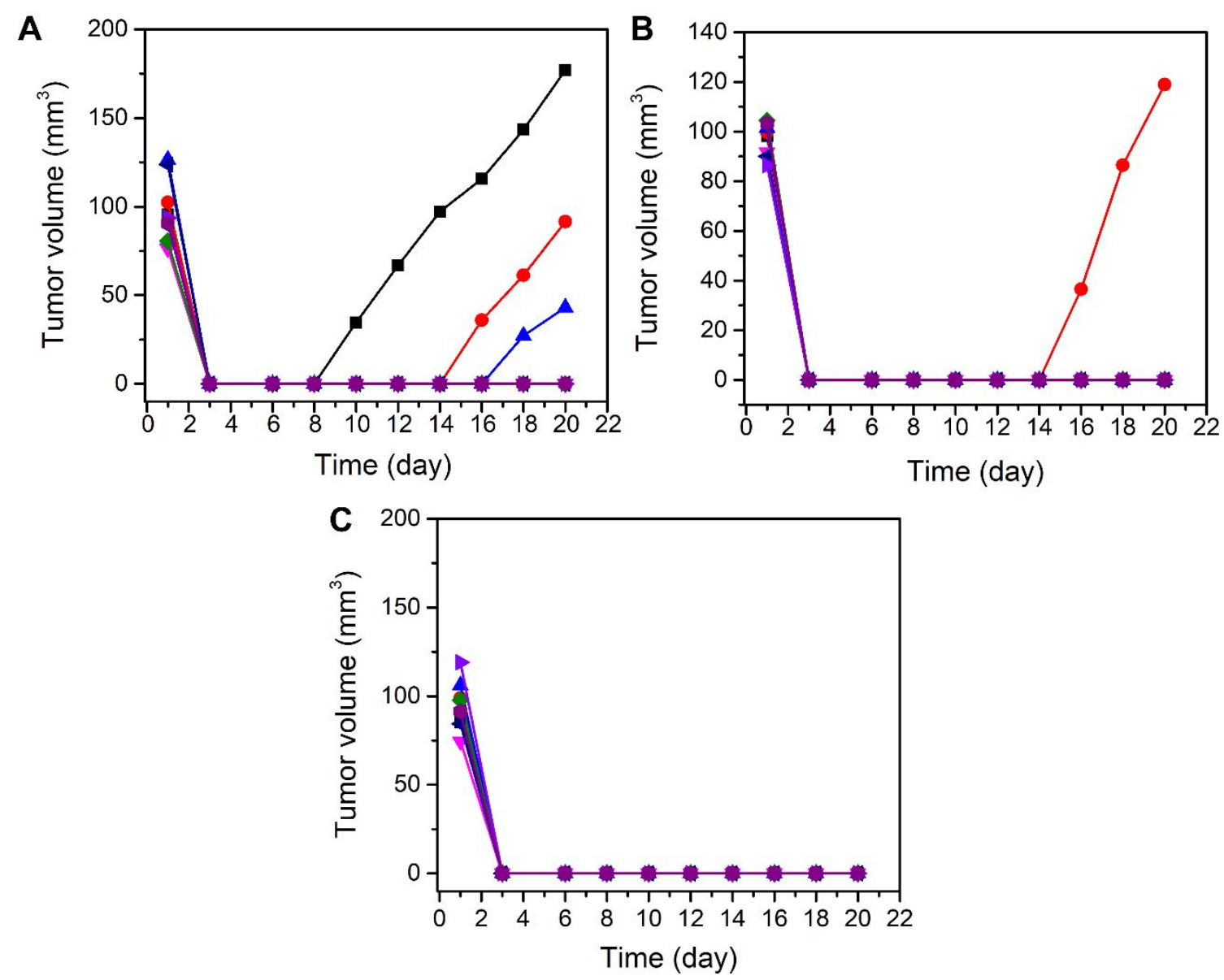

Figure S30. (A) The tumor volume changes in NPs1 $+808 \mathrm{~nm}$ group, (B) The tumor volume changes in NPs2 $+670+808 \mathrm{~nm}$ group, and (C) The tumor volume changes in NPs1 $+670+808 \mathrm{~nm}$ group. 


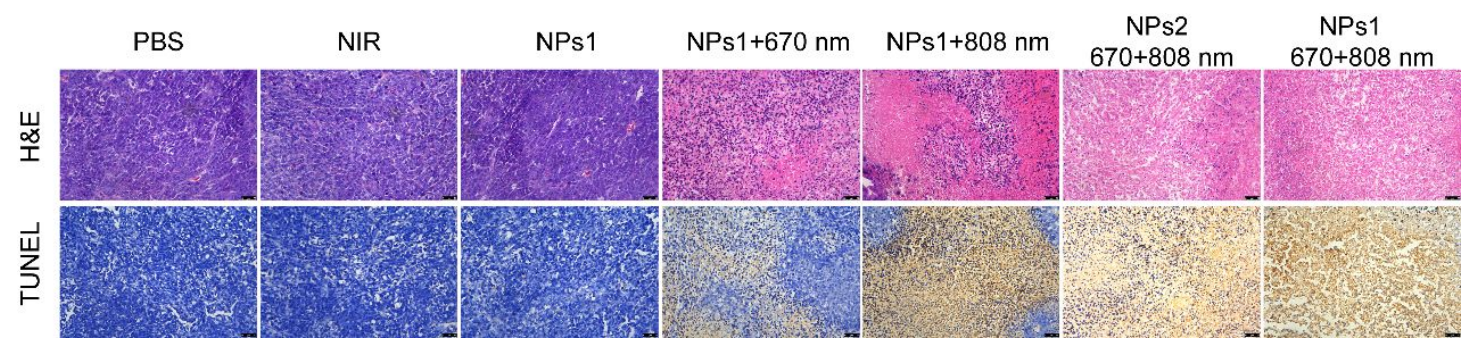

Figure S31. Histopathology analysis (H\&E staining and TUNEL assay) of PC-3 tumor sections on day 24 of the treatment. The tumor sections were obtained on the second day after treatment. NPs1 and NPs2 represent [PHC]PP@HA NPs and [PC]PP@HA NPs, respectively.

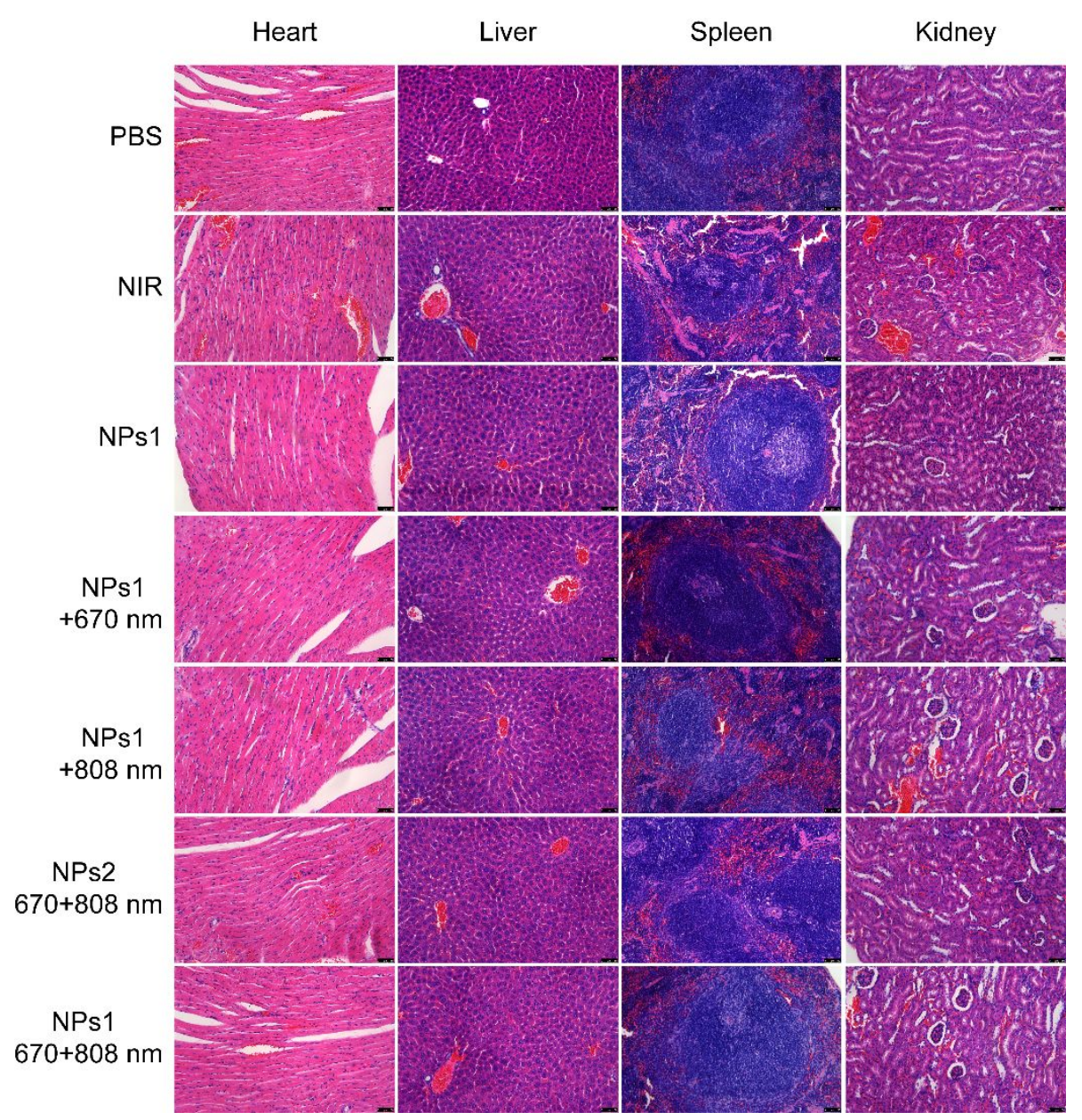

Figure S32. H\&E analysis of main organs after 2 months. NPs1 and NPs2 represent [PHC]PP@HA NPs and [PC]PP@HA NPs, respectively. 

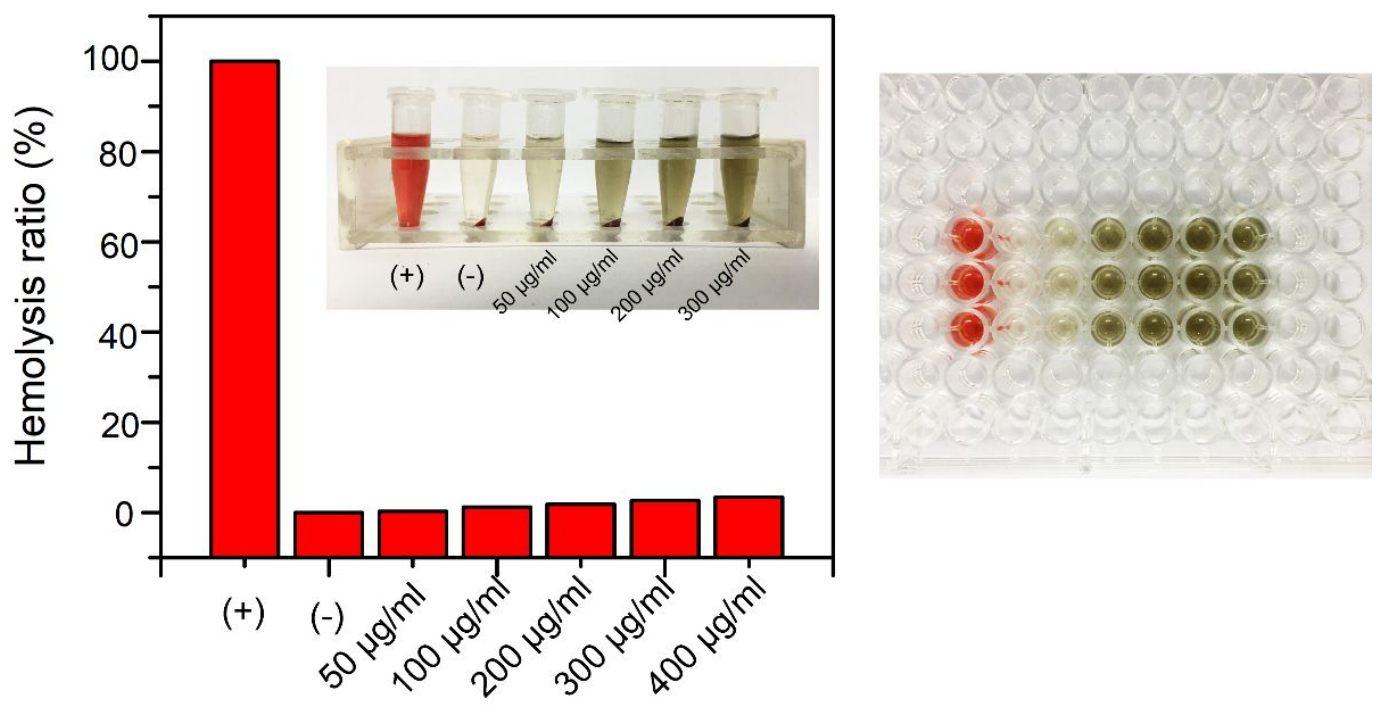

Figure S33. Hemolysis test of [PHC]PP@HA NPs.

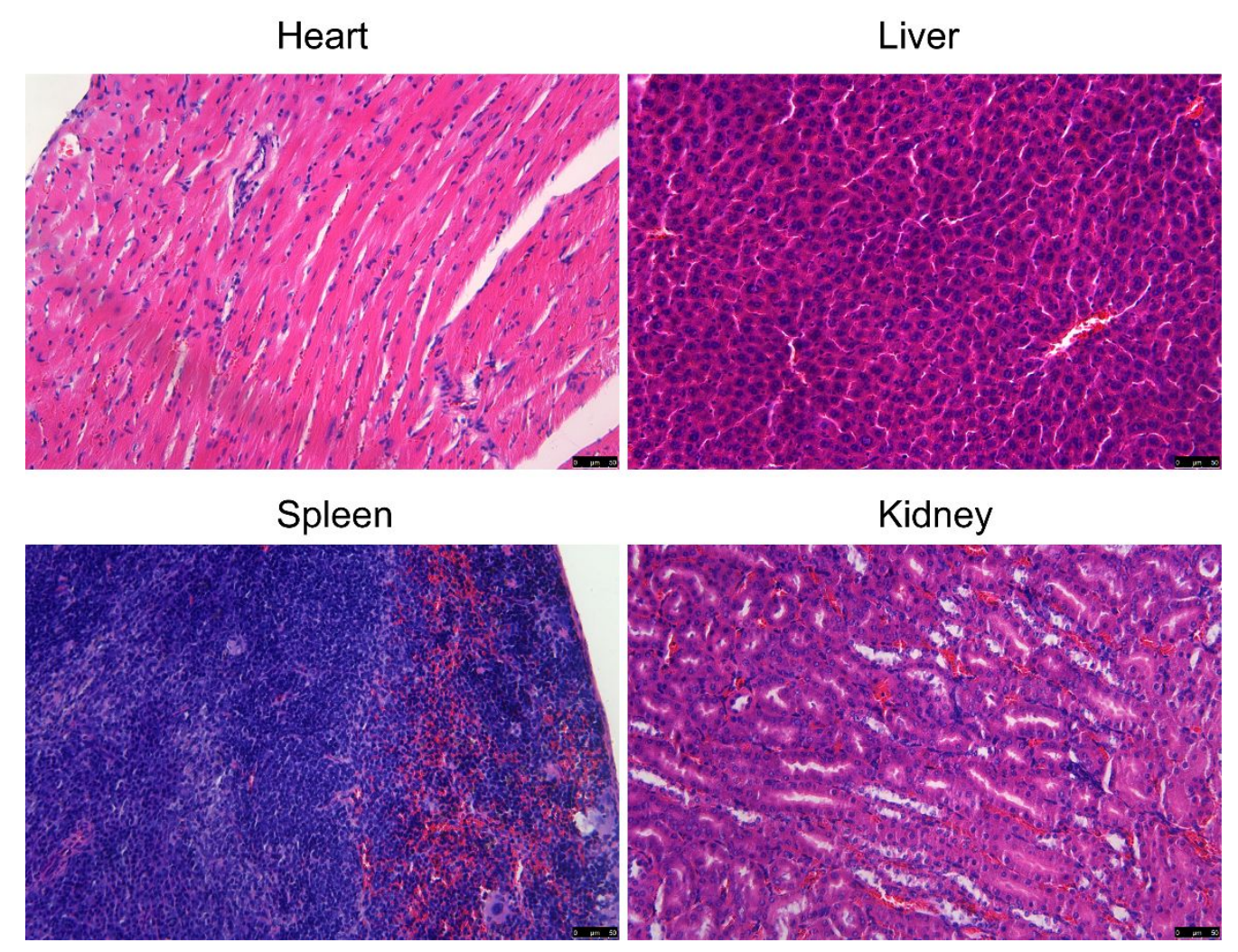

Figure S34. H\&E analysis of main organs in the [PHC]PP@HA NPs group after 2 months. 

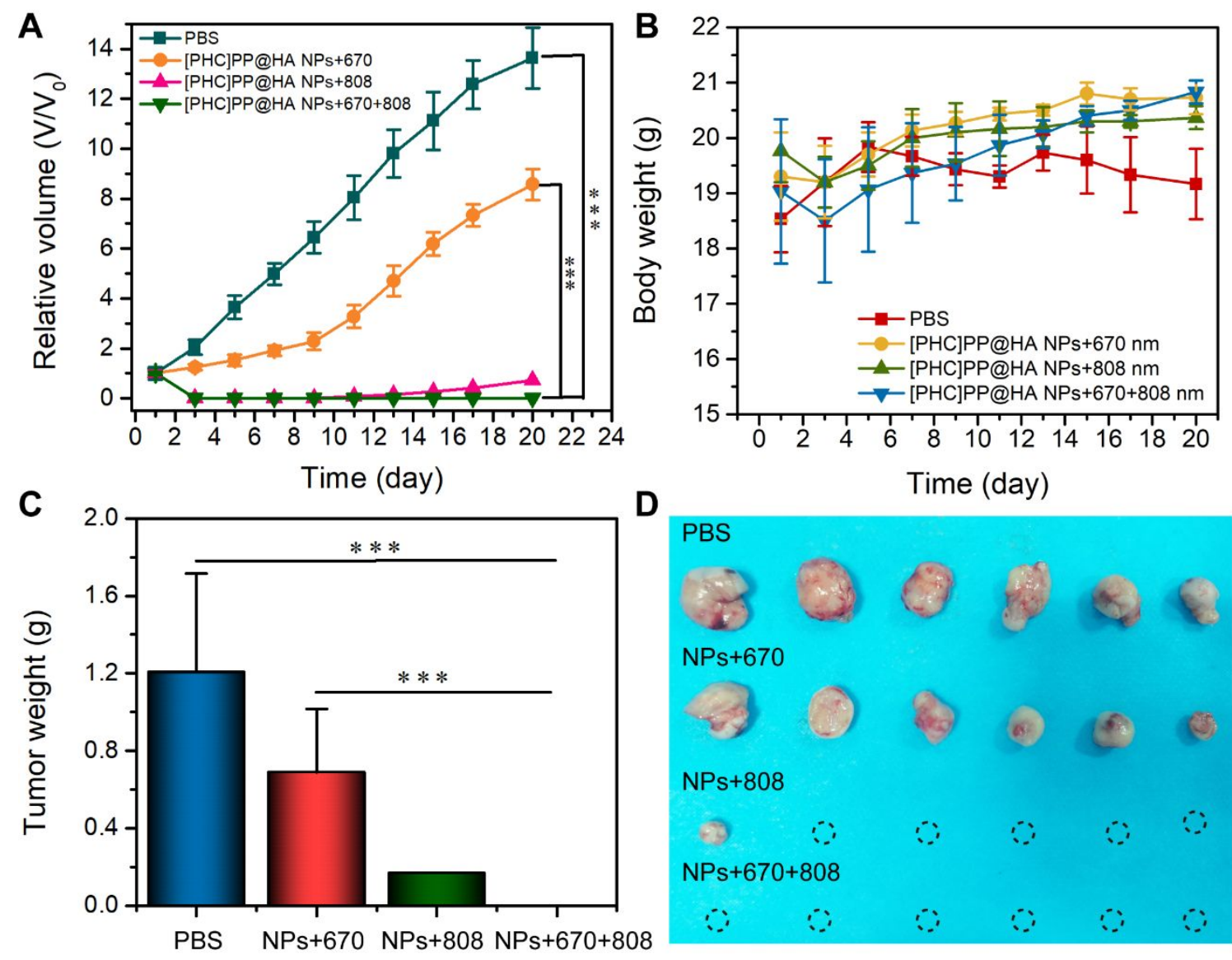

Figure S35. In vivo antitumor performance of [PHC]PP@HA NPs in A549 tumor model. (A) The tumor growth profiles after different treatments $(n=6)$. (B) Body weight of the mice after various treatments. (C, D) Tumor weights and morphology of mice at day 20 after different treatments $(n=6)$. $(* * * P<0.001)$ 


\section{C.Supplementary Table}

Table S1. In vitro cytotoxicities and $\mathrm{IC}_{50}\left(\mu \mathrm{g} \mathrm{mL} \mathrm{m}^{-1}\right)$ of different treatment formulations against PC-3 cells for $48 \mathrm{~h}$ incubation

\begin{tabular}{ccc}
\hline Formulations & $\mathrm{IC}_{50}\left(\mu \mathrm{g} \mathrm{mL}^{-1}\right) /$ Normoxia & $\mathrm{IC}_{50}\left(\mu \mathrm{g} \mathrm{mL}^{-1}\right) /$ Hypoxia \\
\hline PDA+808 nm & 55.49 & 60.57 \\
$\mathrm{Ce} 6+670 \mathrm{~nm}$ & 59.37 & 127.50 \\
$\mathrm{Ce} 6+\mathrm{Hb}+670 \mathrm{~nm}$ & 48.33 & 52.80 \\
$\mathrm{NPs}+670 \mathrm{~nm}+808 \mathrm{~nm}$ & 9.20 & 9.87 \\
\hline
\end{tabular}

Table S2. In vitro cytotoxicities and combination index $\left(\mathrm{CI}_{50}\right)$ of different treatment formulations against PC-3 cells for $48 \mathrm{~h}$ incubation

\begin{tabular}{ccccc}
\hline Formulations & $\begin{array}{c}\mathrm{IC}_{50}\left(\mu \mathrm{g} \mathrm{mL}^{-1}\right) \\
\text { Normoxia }\end{array}$ & $\begin{array}{c}\mathrm{IC}_{50}\left(\mu \mathrm{g} \mathrm{mL}^{-1}\right) \\
/ \text { Hypoxia }\end{array}$ & $\mathrm{CI}_{50} /$ Normoxia & $\mathrm{CI}_{50} /$ Hypoxia \\
\hline $\mathrm{NPs}+670 \mathrm{~nm}$ & 32.30 & 44.38 & - & - \\
$\mathrm{NPs}+808 \mathrm{~nm}$ & 21.84 & 26.74 & - & - \\
$\mathrm{NPs}+670 \mathrm{~nm}+808 \mathrm{~nm}$ & 9.20 & 9.87 & 0.71 & 0.59 \\
\hline
\end{tabular}

Table S3. The statistic analysis of the apoptosis rate of tumor cells in different groups.

\begin{tabular}{cccccccc}
\hline & PBS & Laser & NPs1 & $\begin{array}{c}\text { NPs1+ } \\
670 \\
\mathrm{~nm}\end{array}$ & $\begin{array}{c}\text { NPs1+ } \\
808 \\
\mathrm{~nm}\end{array}$ & $\begin{array}{c}\text { NPs2+ } \\
670+808 \\
\mathrm{~nm}\end{array}$ & $\begin{array}{c}\text { NPs1+ } \\
\mathrm{nm}\end{array}$ \\
\hline $\begin{array}{c}\text { Area fraction of } \\
\text { apoptotic nuclei (\%) }\end{array}$ & 1.07 & 0.97 & 1.04 & 11.97 & 17.97 & 14.83 & 11.69 \\
$\begin{array}{c}\text { Area fraction of total } \\
\text { nuclei (\%) }\end{array}$ & 27.21 & 27.30 & 25.45 & 42.68 & 34.95 & 16.70 & 12.03 \\
$\begin{array}{c}\text { Apoptotic rate (\%) } \\
\text { Apos }\end{array}$ & 3.93 & 3.55 & 4.09 & 28.05 & 51.42 & 88.80 & 97.17 \\
\hline
\end{tabular}

